ARTICLE

https://doi.org/10.1038/s41467-018-07359-8

\title{
Therapeutic faecal microbiota transplantation controls intestinal inflammation through IL10 secretion by immune cells
}

\author{
Claudia Burrello (1,2 ${ }^{1,2}$ Federica Garavaglia', Fulvia Milena Cribiù ${ }^{3}$, Giulia Ercoli³, Gianluca Lopez ${ }^{3}$, \\ Jacopo Troisi ${ }^{4,5,6}$, Angelo Colucci ${ }^{4,5}$, Silvia Guglietta1 ${ }^{1}$, Sara Carloni ${ }^{7}$, Simone Guglielmetti ${ }^{8}$, Valentina Taverniti ${ }^{8}$, \\ Giulia Nizzoli ${ }^{9}$, Silvano Bosari (iD ${ }^{3}$, Flavio Caprioli ${ }^{9}, 10$, Maria Rescigno ${ }^{7}$ \& Federica Facciotti (i) ${ }^{1}$
}

Alteration of the gut microbiota has been associated with different gastrointestinal disorders. Normobiosis restoration by faecal microbiota transplantation (FMT) is considered a promising therapeutic approach, even if the mechanisms underlying its efficacy are at present largely unknown. Here we sought to elucidate the functional effects of therapeutic FMT administration during experimental colitis on innate and adaptive immune responses in the intestinal mucosa. We show that therapeutic FMT reduces colonic inflammation and initiates the restoration of intestinal homeostasis through the simultaneous activation of different immune-mediated pathways, ultimately leading to IL-10 production by innate and adaptive immune cells, including CD4 ${ }^{+}$T cells, iNKT cells and Antigen Presenting Cells (APC), and reduces the ability of dendritic cells, monocytes and macrophages to present $\mathrm{MHCII-}$ dependent bacterial antigens to colonic $T$ cells. These results demonstrate the capability of FMT to therapeutically control intestinal experimental colitis and poses FMT as a valuable therapeutic option in immune-related pathologies.

\footnotetext{
${ }^{1}$ Department of Experimental Oncology, European Institute of Oncology IRCCS, via Adamello 16, Milan 20139, Italy. ${ }^{2}$ Department of Oncology and Hematooncology, Università degli Studi di Milano, via F. Sforza 28, Milan 20122, Italy. ${ }^{3}$ Pathology Unit, Fondazione IRCCS Cà Granda, Ospedale Maggiore Policlinico, via F. Sforza 35, Milan 20135, Italy. ${ }^{4}$ Department of Medicine, Surgery and Dentistry, "Scuola Medica Salernitana", University of Salerno, Baronissi 84081 SA, Italy. ${ }^{5}$ Theoreo srl, Spin-off company of the University of Salerno, Via degli Ulivi 3, 84090 Montecorvino Pugliano, SA, Italy. ${ }^{6}$ European Biomedical Research Institute of Salerno (EBRIS), Via S. de Renzi, 3, 84125 Salerno, SA, Italy. ${ }^{7}$ Laboratory of Mucosal Immunology and Microbiota, Humanitas Clinical and Research Center, Via Manzoni 56, Milan 20089, Italy. ${ }^{8}$ Department of Food Environmental and Nutritional Sciences (DeFENS), Università degli Studi di Milano, Milan 20133 via Celoria 2, Italy. ${ }^{9}$ Gastroenterology and Endoscopy Unit, Fondazione IRCCS Cà Granda, Ospedale Maggiore Policlinico, via F. Sforza 35, Milan 20135, Italy. ${ }^{10}$ Department of Pathophysiology and Transplantation, Università degli Studi di Milano, via F. Sforza 28, Milan 20135, Italy. Correspondence and requests for materials should be addressed to F.F. (email: Federica.facciotti@ieo.it)
} 
T he gut mucosa constitutes a unique environment exposed to more than $10^{14}$ commensal bacteria, which establish a mutualistic relationship with the host, providing metabolic functions and contributing to shape the immune system ${ }^{1}$. Maintenance of intestinal homeostasis requires several methods to physically confine commensal bacteria to the intestinal lumen, while keeping the full capability to control colonization by pathogenic bacteria ${ }^{1}$. Variations of this equilibrium induce the recruitment and expansion of several immune cell types contributing to initiate and propagate intestinal inflammation, or to restore homeostasis by activating tolerogenic mechanisms ${ }^{2}$.

Alteration in the composition of the gut microbiota (dysbiosis) has been associated with a wide range of gastrointestinal diseases, including recurrent $C$. difficile infection $(\mathrm{CDI})^{3}$, inflammatory bowel diseases (IBD, Crohn's disease, $\mathrm{CD}$, and Ulcerative colitis, $\mathrm{UC})^{4,5}$ and colorectal cancer (CRC) ${ }^{6}$.

Current theories suggest that IBD onset is secondary to an exaggerated reaction of gut-associated lymphoid tissue against the luminal microbiota ${ }^{7}$. Whether this is a primary defect or it is secondary to intestinal dysbiosis is still debated. Indeed, a reduced biodiversity in both mucus-associated and faecal bacterial communities has been observed both in IBD patients and in their first degree relatives ${ }^{4,8,9}$. Moreover, IBD patients showed reduced diversity of their gut microbiome, expansion of pro-inflammatory bacteria like Enterobacteriaceae and Fusobacteriaceae and depletion of phyla with anti-inflammatory functional properties such as Firmicutes ${ }^{10}$. Additionally, changes in mucus-associated microbiota have been observed in the ileal mucosa of children with treatment-naïve IBD, independently from intestinal inflammation ${ }^{4}$. These observations suggest that intestinal dysbiosis may be causally related and might precede mucosal inflammation in IBD patients. This has also been confirmed by the induction of experimental inflammation upon transfer of dysbiotic microbiota or single commensal bacterial species in germ-free mice ${ }^{11}$.

Intestinal epithelial damage has been proposed to be a key event favouring bacteria translocation out of the intestinal lumen, thus facilitating the recognition of antigens derived from the dysbiotic microflora by pathogenic $\mathrm{T}$ cells in IBD patients ${ }^{7}$. A complex physical barrier composed by a monolayer of polarized epithelial cells has been developed to avoid exposure to the intestinal microbiome ${ }^{12}$. Additionally, to prevent the bacterial access to epithelial cells, mucous layers of different densities line on top of the epithelium. Other host's cellular components contribute to homeostasis maintenance through the secretion of antimicrobial peptides (AMPs) ${ }^{12}$.

Manipulation of the intestinal microbiome is becoming a therapeutic option in several gastrointestinal disorders ${ }^{13}$. Faecal microbiota transplantation (FMT), i.e. the infusion of healthy donor faeces in the gut of a recipient to treat a disorder associated with microbiota alterations, is receiving great consideration thanks to its effectiveness against refractory and recurrent $\mathrm{CDI}^{14,15}$. Increasing evidences from recent Randomized Clinical Trials (RCTs) are also supporting the possibility to utilise FMT to treat mild-to-moderate UC patients ${ }^{16-18}$. Despite its recent successes, it is still largely unknown how FMT functionally modulates the intestinal immune system. Very limited data are available on the therapeutic re-equilibration of the gut microbiota in murine models of intestinal inflammation ${ }^{19,20}$.

Since the intestinal immune system is sensitive to variations of luminal and mucus-associated bacteria, we postulated that therapeutic FMT administered during intestinal inflammation might directly modulate both innate and adaptive mucosal immune responses towards the control of intestinal inflammation. Given the predominant role of $\mathrm{CD} 4^{+} \mathrm{T}$ cells in orchestrating IBDrelated inflammatory processes ${ }^{21}$, largely due to their differentiation into IFN $\gamma / \mathrm{IL}$-17-secreting effector subtypes, we focused our primary analysis on the functional variations occurring in these cell populations upon FMT. The effect of microbiota alterations during colitis induction and upon FMT treatment in colitogenic mice was additionally assessed on iNKT cells, a subset of $\alpha \beta-T$ cells recognizing both self- and microbial-derived glycolipids ${ }^{22,23}$ and heavily influenced by bacterial-derived antigens in early life ${ }^{24,25}$. We also recently showed that colonic iNKT cells are sensitive to microbiota alterations occurring after short term antibiotic treatment ${ }^{26}$. To selectively track this rare cell subset, we took advantage of a novel reporter mouse strain, the CXCR6 ${ }^{\text {EGFP }}$ mouse ${ }^{27}$.

Here we demonstrate that therapeutic administration of FMT during experimental colitis exerts beneficial effects, which are associated to the simultaneous activation of several antiinflammatory pathways. Upon FMT, variations in the intestinal ecology towards IL-10-inducing microbial communities and the production of tolerogenic IL-10 by mucosal innate and adaptive cell subsets altogether concur to resolve the inflammation.

\section{Results}

Therapeutic FMT reduces inflammation in DSS-induced colitis. CXCR6-EGFP/+ mice ${ }^{27}$ are susceptible to DSS-induced colitis (Supplementary Fig. 1) and can be used to track intestinal CD4 ${ }^{+}$ $\mathrm{T}$ cell subsets, including the relatively rare iNKT cells (Supplementary Fig. 2), under homeostatic conditions ${ }^{26}$ and during experimental intestinal inflammation.

Upon establishment of DSS-induced acute colitis, the functional effects of therapeutic FMT were evaluated. Mucus and faeces derived from normobiotic mice were collected and microbiota transfer was performed in colitic mice by oral gavage for 3 consecutive days according to the scheme described in Fig. 1a. At sacrifice, FMT-treated mice showed reduced signs of intestinal inflammation, as indicated by decreased weight loss (Fig. 1b) and increased colonic length (Fig. 1c and Supplementary Fig. 3a). These effects were associated to an amelioration of the intestinal inflammation measured by the histological score upon FMT (Fig. 1d, e and Supplementary Fig. 3b). Moreover, in agreement with previously published reports ${ }^{19}$, FMT reduced the expression of the pro-inflammatory Il1 $\beta$ (Fig. If and Supplementary Fig. 3c) in the colonic mucosa.

To evaluate whether FMT might exert protective effects on gut barrier functions, the colonic expression of antimicrobial peptides and mucins was also tested (Fig. 1g, h). The expression of the tight junction protein Zo-1 was not affected upon FMT treatment in the colons of colitic mice (Supplementary Fig. 3d). On the contrary, Camp and S100A8, two antimicrobial peptides playing anti-inflammatory roles during acute intestinal inflammation ${ }^{28}$ were upregulated upon FMT administration (Fig. 1g). Similarly, a tendency toward upregulation of Mucl and Muc4, two mucins exerting anti-inflammatory functions in response to pathogens ${ }^{29}$, and a downregulation of Muc3 were observed by FMT treatment in acute DSS-colitis (Fig. 1h).

Thus, our results demonstrate that FMT therapeutic administration during experimental acute colitis ameliorates intestinal inflammation.

FMT induces variations in the microbial communities. Next, variations in the microbial communities in colitic mice treated or not with FMT were evaluated. Fecal samples of DSS and DSS + FMT mice were collected at day 11 post colitis induction and subjected to microbiome profiling using $16 \mathrm{~S}$ rRNA gene sequencing on the Illumina MiSeq platform. An unweighted UniFrac-based comparison of the microbiota isolated from untreated, DSS and DSS + FMT-treated recipient mice was 
a

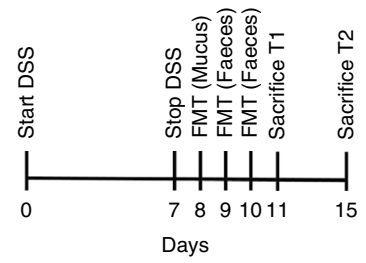

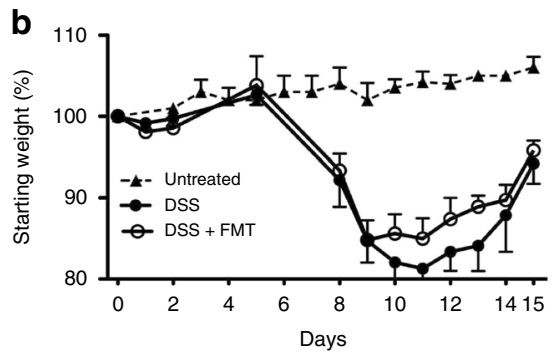

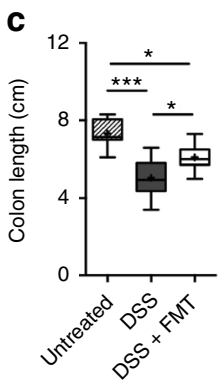

d

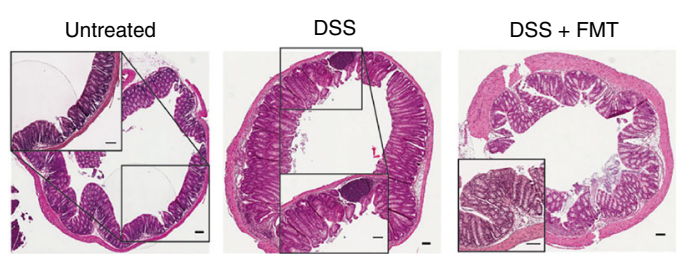

e

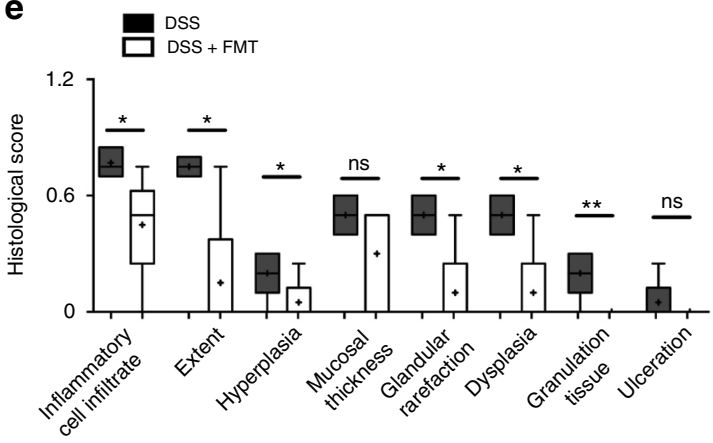

f

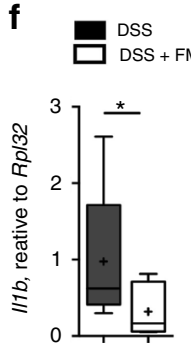

g

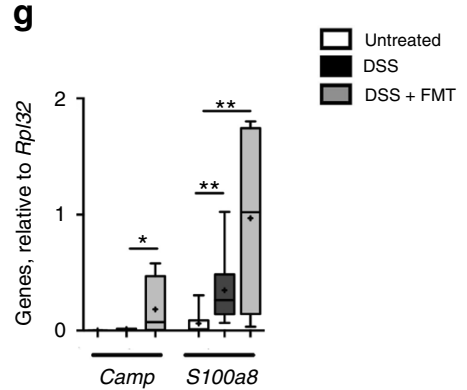

h

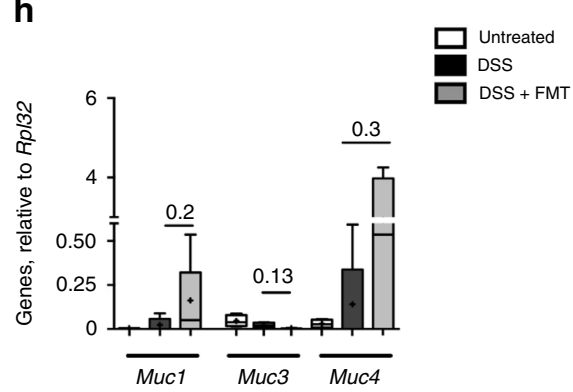

Fig. 1 Therapeutic FMT ameliorates DSS-induced experimental colitis. a Schematic representation of FMT treatment during acute DSS experimental colitis. b, c Weight loss (b) and colon length (c) of untreated (triangles and striped boxes), colitic (black dots and black boxes), or colitic mice treated with FMT (white dots and white boxes). d, e H\&E staining (scalebar $100 \mu \mathrm{m}$ ) and cumulative histological score of colon specimens obtained from DSS-treated and FMT-treated mice; (e) Detailed histological evaluation of mice with decreased histological score (white boxes) compared to DSS-treated mice (black boxes). $\mathbf{f}$ Colonic expression levels of $I 11 \beta$ in colitic (black boxes) and FMT-treated (white bars) mice. $\mathbf{g}$, h Colonic expression levels of Camp, S100A8 (g) and Muc1, Muc3, Muc4 (h) in untreated (white boxes), DSS treated (black boxes) or DSS + FMT-treated (gray boxes) mice. Statistical significance was calculated using a Mann-Whitney test for comparison within two groups or Kruskal-Wallis test with Dunn's multiple comparison correction within more than two groups. ${ }^{\star} P<0.05,{ }^{\star \star} P<0.01,{ }^{\star \star \star} P<0.001,{ }^{\star \star \star \star} P<0.0001$ were regarded as statistically significant. Outliers were detected with Grubb's test. Non parametric distributions were represented as median $+/$ - interquartile range. In Box and whiskers plots, centre line represents median; cross, represents mean. In (b) UT $n=10$, DSS $n=10$, DSS + FMT $n=10 . \ln (\mathbf{c})$ UT $n=12$, DSS $n=12$, DSS + FMT $n=14 . \ln (\mathbf{d})$ UT $n=8$, DSS $n=15$, DSS + FMT $n=19$. In (f-h) UT $n=5$, DSS $n=6$, DSS + FMT $n=7$

performed (Fig. 2a and Supplementary figure 4a). Principle component analysis (PCA) differentiated untreated mice from the experimental groups (DSS and DSS + FMT). The intestinal microbiota of recipient mice receiving or not a FMT did not macroscopically differ at sacrifice, possibly due to similar relative abundances of the top 10 most abundant species among DSS and DSS + FMT-derived samples (Fig. 2b). Similarly, the microbiota isolated from DSS-treated mice did not show a lower a-diversity when compared to DSS + FMT-derived microbiota, as reflected by the Chaol and Shannon indexes (Fig. 2c).

Nonetheless, a detailed phylogenetic analysis of the taxonomic composition of the microbiome of colitic mice treated or not with FMT showed that the reduced inflammatory conditions observed upon FMT administration were associated, with variations in the abundance of specific taxa, including Firmicutes and Verrucomicrobiae $^{30,31}$. (Fig. 2d-f).
Significant changes towards restoration of normobiosis were detected among the less abundant families belonging to the Firmicutes phylum in the DSS + FMT-derived microbiota. For instance, Clostridiaceae and Clostridiales, which were expanded in colitic mice, were reduced upon FMT and returned to levels comparable to those observed in untreated mice (Fig. 2e).

FMT-derived samples showed significant increases of known commensals used in probiotics preparations ${ }^{32}$, including Lactobacillaceae and Streptococcus sp., and of the SCFA-producing taxa Erysipelotrichaceae, Ruminococcaceae, Odoribacter and Olsenella (Fig. 2f and Supp Fig. 4B), which are reported to be reduced in IBD patients ${ }^{4,33}$.

Of note, metabolomic analysis showed an increased faecal content of complex sugars including lactose and maltose in DSStreated mice, a possible consequence of impaired digestion or defective intestinal absorption ${ }^{34}$, whose levels were normalized by 
a
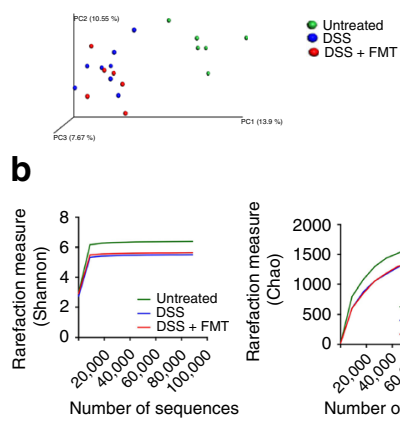

e

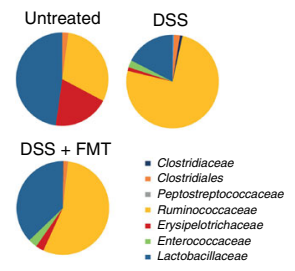

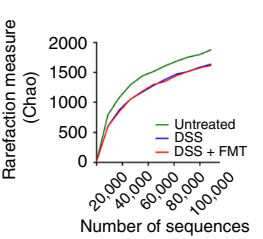

f

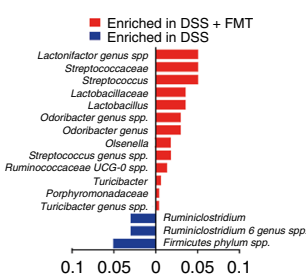

C

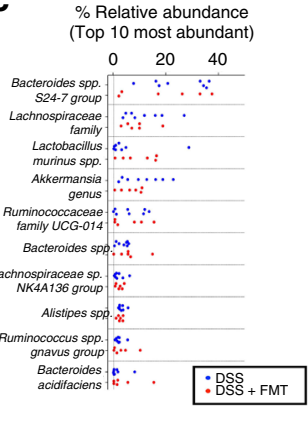

d
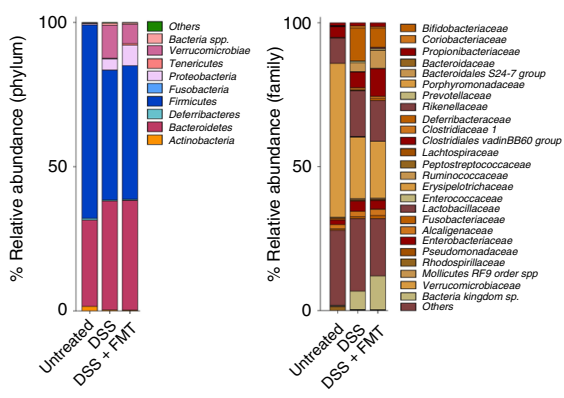

$\mathbf{g}$

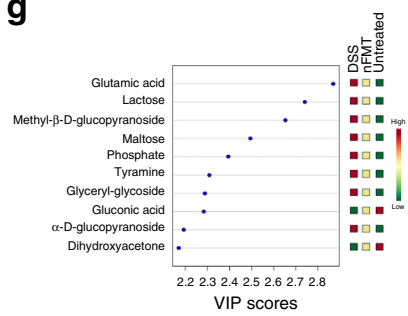

h

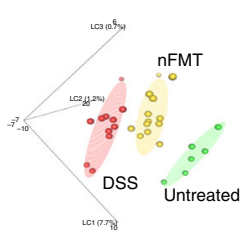

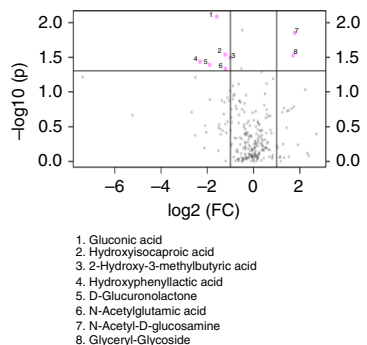

Fig. 2 Gut microbiota analysis upon FMT treatment in colitic mice. a Microbiome clustering based on unweighted Principal Coordinate Analysis (PCA) UniFrac metrics of fecal gut microbiota derived from DSS treated (blue dots), DSS + FMT-treated (orange dots) and untreated (red dots) mice. b Relative abundance of the top 10 most abundant OTUs in DSS (blue) and DSS + FMT (red) treated mice. c Rarefaction curves showing microbial richness based on the Chao1 index (bottom panel) and microbial richness and evenness on the Shannon index (upper panel). Green lines, untreated, blue lines, DSS treated; red lines, DSS + FMT-treated samples. d Bar plots of the taxonomic composition showing relative abundances $>1 \%$ of bacterial phyla ( $d$, left panel) and families (d, right panel). e Pie charts showing the relative abundance of the less abundant families belonging to the Firmicutes phylum. $\mathbf{f}$ Comparison of the relative abundances of different taxa between DSS (blue) and DSS + FMT (red) treated mice. Statistical significant difference was assessed through Oneway ANOVA with LSD post-hoc test ${ }^{\star} p<0.05,{ }^{\star \star} p<0.01 . \mathbf{g}$, $\mathbf{h}$ Partial Least square-discrimination analysis (PLSD-DA, g) and volcano plot (h) on metabolomics data ( $g$, right panel) and heat map of metabolites that have contributed most to class separation ( $g$, left panel) of faecal samples of untreated, DSS and DSS + FMT-treated mice. Statistical significant difference was assessed through Mann-Whitney. In (a-f) UT $n=6$, DSS $n=8$, DSS + FMT $n=6 . \ln (\mathbf{g}, \mathbf{h})$ UT $n=6$, DSS $n=11$, DSS + FMT $n=13$

FMT-treatment. Similarly, glutamic acid, a metabolite altered in IBD patients ${ }^{35}$, decreased upon FMT while gluconic acid and Dihydroxiacetone, involved in natural detoxification activities ${ }^{36}$, increased upon FMT (Fig. 2g, h).

Altogether, these findings suggest that the beneficial effects of FMT during intestinal inflammation are associated with a reshuffling of the microbiota communities towards restoration of functional normobiosis.

FMT effects rely on the presence of normobiotic ecologies. Once documented the beneficial effects of FMT, the impact of the donor microbiota composition on the resolution of intestinal inflammation was evaluated.

To do so, mucus and faecal samples were obtained from normobiotic or dysbiotic mice, i.e from healthy mice left untreated or treated for 7 days with DSS, and FMT was performed in colitic mice as previously described in Fig. 1a.

Relevant differences between the two types of donors used for FMT experiments were confirmed by metagenomic analyses (Fig. 3a, b and Supplementary fig 5A). As previously shown ${ }^{31}$, the microbiota of dysbiotic mice was characterized by a contraction of Bacteroidales S24-7, Lachnospiraceae and Bifidobacteriaceae and an expansion of Enterobacteriaceae and Bacteriaceae as compared to that of normobiotic mice (Fig. 3a).

As a consequence, an amelioration of the intestinal inflammation was reported in mice receiving a normobiotic FMT but not in those receiving a dysbiotic FMT, as demonstrated by an increase in colon length (Fig. 3c) and a decrease, albeit not significant, in the expression levels of colonic pro-inflammatory $I l l b$ and $\operatorname{Tnf}$ (Fig. 3d).

Consistently, the microbial composition of recipient mice that were transplanted with a dysbiotic FMT was also more similar to that of DSS-treated mice, enriched in pathobionts such as E.Coli/ Shigella (Fig. 3e), while the microbiota of mice receiving a normobiotic FMT were more similar to that of untreated mice, enriched in protective SCFA-producing bacteria (Fig. 3e). Similarly, metabolomic analysis of the faeces of recipient mice treated with normobiotic FMT revealed the presence of metabolites associated to scavenging of free radicals (d-glucunolactone) and metals (gluconic acid) ${ }^{36}$, to the control of ROS production and neutrophils activity (hydroxiphenillactic acid) and chemotaxis (LPA) ${ }^{37}$, and to SCFA production (Valeric acid) ${ }^{38}$ (Fig. 3f).

Next, we evaluated if normobiotic donors of different origins might be equally capable to control intestinal inflammation when transplanted into colitic mice. Mucus and faecal samples were isolated form age- and sex-matched $\mathrm{C} 57 \mathrm{Bl} / 6$ mice obtained from different sources, i.e. from two commercial animal vendors (Charles River srl and Envigo srl) and from in-house bred C57Bl/ 6 wild-type colony (IEO animal facility). Interestingly, the FMT performed with the microbiota isolated from the different normobiotic donors was equally capable to control intestinal inflammation, as shown by similar colon length (Fig. 4a), 
a

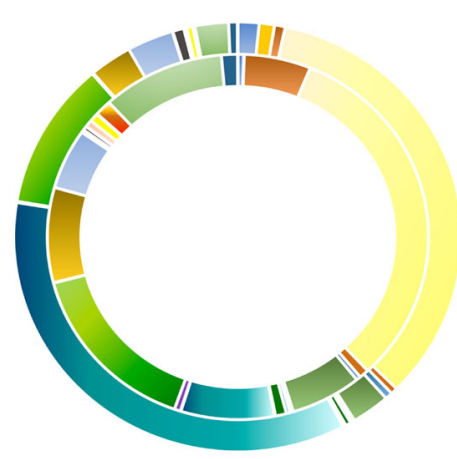

Normobiotic (out) dysbiotic (in)
Bifidobacteriaceae

Coriobacteriaceae

Bacteroidaceae

Bacteroidales S24-7 group

- Porphyromonadaceae

- Prevotellaceae

- Rikenellaceae

- Clostridiales vadin BB60 group

- Lachnospiraceae

- Peptostreptococcaceae

- Ruminococcaceae

- Erysipelotrichaceae

- Lactobacillaceae

- Alcaligenaceae

Rhodospirillaceae

- Mollicutes RF9 order spp.

- Verrucomicrobiaceae

- Bacteria kingdom spp. b

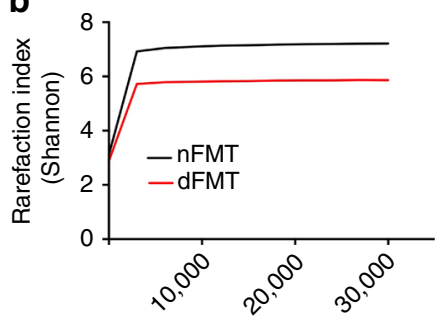

Number of sequences

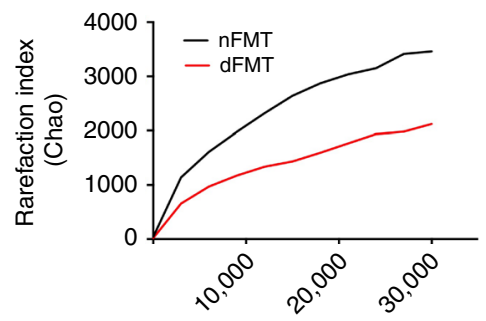

Number of sequences

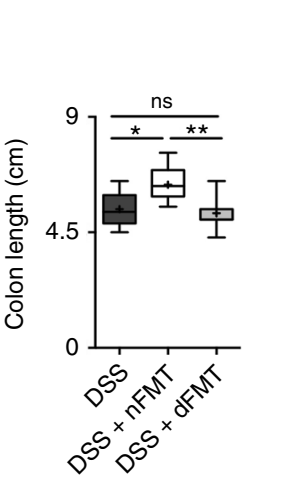

f e

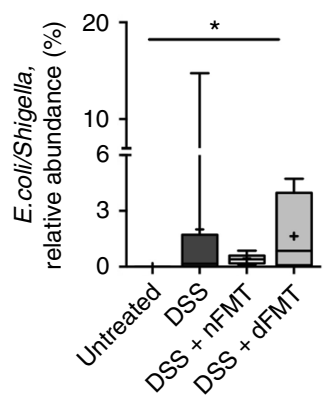

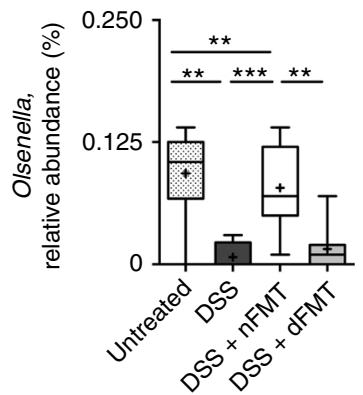

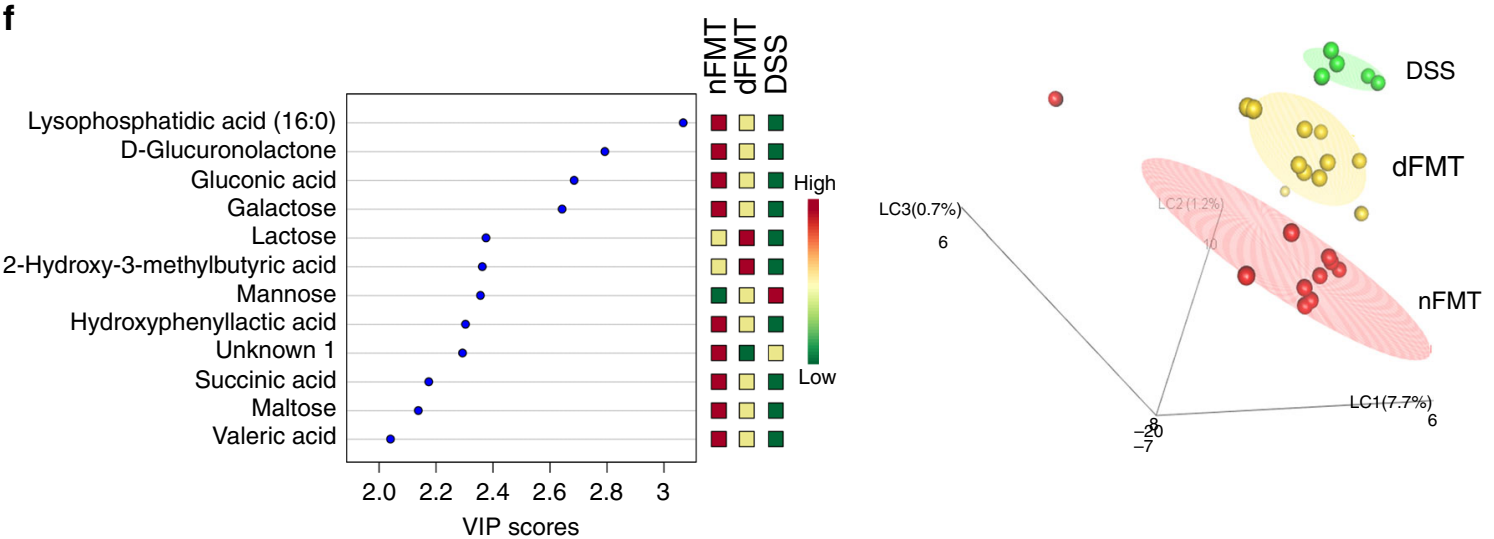

Fig. 3 FMT effects upon transfer of normobiotic or dysbiotic ecologies. a Comparison of relative abundancies of different taxa between normobiotic (outer chart) and dysbiotic (inner chart) faecal microbiota. b Rarefaction curves showing microbial richness based on the Chao1 index (right panel) and microbial richness and evenness on the Shannon index (left panel) Black curves, normal FMT samples, red curves, dysbiotic FMT samples. c Colon length of DSS (black boxes), DSS + nFMT (white boxes) or DSS + dFMT (gray boxes) treated mice. d Colonic expression of Tnf and II1b in DSS (black boxes), DSS + nFMT (white boxes) or DSS + dFMT (gray boxes) treated mice. e Relative abundance of E.Coli/Shigella (left panel) and Olsenella (right panel) in untreated (dotted white boxes), DSS treated (black boxes), DSS + nFMT (white boxes) and DSS + dFMT (gray boxes) mice. f Partial Least square-discrimination analysis (PLSD-DA) on metabolomics data (right panel) and heat map of metabolites that have contributed most to class separation (left panel) of faecal samples of DSS, DSS + nFMT and DSS + dFMT treated mice. Statistical significance was calculated using a Mann-Whitney test for comparison within two groups or Kruskal-Wallis test with Dunn's multiple comparison correction within more than two groups. ${ }^{\star} P<0.05,{ }^{\star \star} P<0.01,{ }^{\star \star \star} P<0.001$ were regarded as statistically significant. Outliers were detected with Grubb's test. Non parametric distributions were represented as median $+/-$ interquartile range. In Box and whiskers plots, centre line represents median; cross, represents mean. $\ln (\mathbf{a}, \mathbf{b}) \mathrm{nFMT} n=7, \mathrm{dFMT} n=7 . \ln (\mathbf{c}, \mathbf{d}) \mathrm{DSS} n=7, \mathrm{DSS}+\mathrm{nFMT} n=13$, DSS + dFMT $n=7$. In (e) UT $n=6$, DSS $n=8$, DSS + nFMT $n=7$, DSS + dFMT $n=7$. In (f) DSS $n=5$, DSS + nFMT $n=10$, DSS + dFMT $n=11$ 

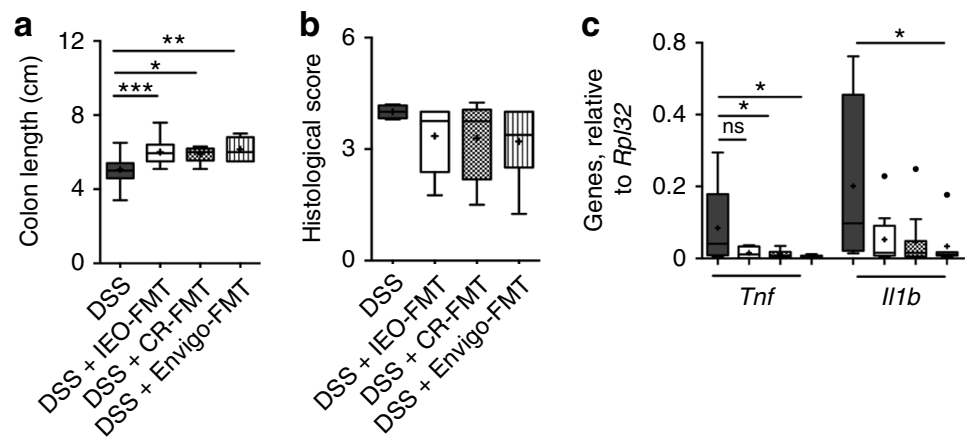

d

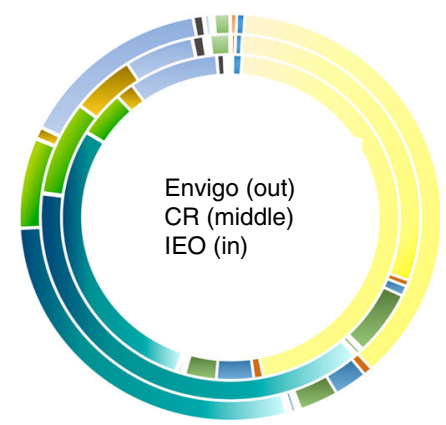

- Bifidobacteriaceae

Ruminococcaceae

- Bacteroidaceae

- Erysipelotrichaceae

Bacteroidales S24-7 group

- Porphyromonadaceae

- Enterococcaceae

- Prevotellaceae

- Lactobacillaceae

- Alcaligenaceae

- Rikenellaceae

Enterobacteriaceae

- Clostridiales vadin BB60 group

- Lachnospiraceae

Verrucomicrobiaceae

- Bacteria kingdom spp. e

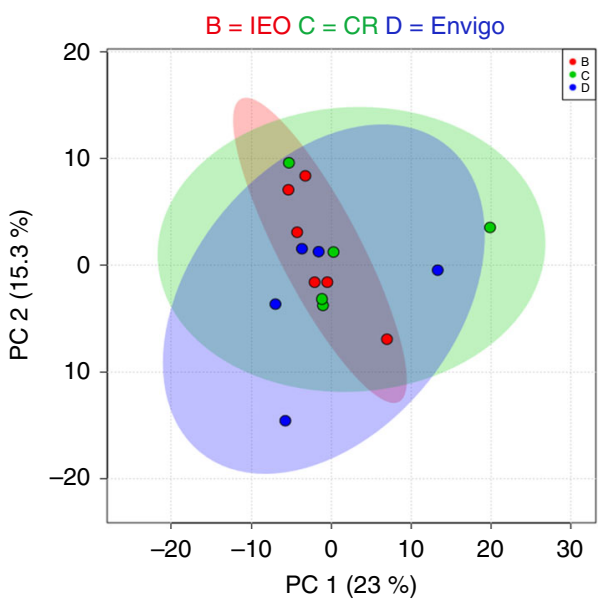

f

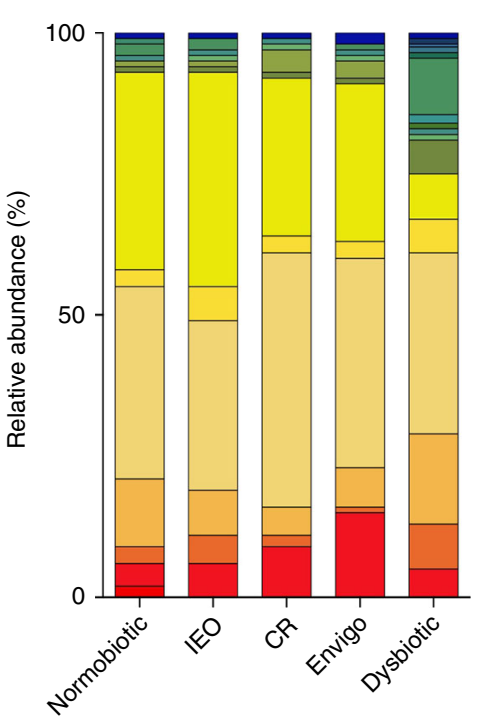

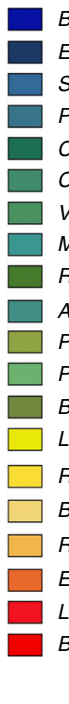

Bacteria kingdom spp. Enterobacteriaceae Streptococcaceae

Peptostreptococcaceae

Clostridiales vadin BB60 group

Coriobacteriaceae

Verrucomicrobiaceae

Mollicutes RF9 order spp.

Rhodospirillaceae

Alcaligenaceae

Prevotellaceae

Porphyromonadaceae

Bacteroidaceae

Lachnospiraceae

Rikenellaceae

Bacteroidales S24-7 group

Ruminococcaceae

Erysipelotrichaceae

Lactobacillaceae

Bifidobacteriaceae

histological score (4b) and expression of colonic proinflammatory genes (Fig. 4c)

As previously observed ${ }^{11,39,40}$, donor mice sharing the same genetic background, sex and age, but raised in different animal facilities harbored a microbiota genetically similar, not identical, (Fig. 4d, and Supplementary fig 5b) but capable to perform overlapping metabolic activities (Fig. 4e). In accordance to very recent data ${ }^{41}, 90$ to $93 \%$ of the total taxa relative abundance of normobiotic mice, regardless of its origin, was composed by a similar core microbial ecology of Bacteroidales S24-7 (30-45\% among groups), Lachnospiraceae (28-38\%), Lactobacillaceae (6-15\%), Ruminococcaceae (5-8\%), Rikenellaceae (3-6\%), Bifidobacteriaceae (1-2\%) and Erysipelotrichaeceae (1-5\%), that in dysbiotic mice accounted only for a total $75 \%$ (Fig. $4 \mathrm{f}$ ). These taxa have been functionally associated to homeostatic metabolic activities, including SCFA production leading to Treg cells differentiation and IL-10-production ${ }^{42-44}$. On the contrary, the taxa expanded in dysbiotic mice accounting for a $25 \%$ of the total 
Fig. 4 Similar ecologies from different FM donors elicit same beneficial effects. a, b Colon length (a) and cumulative histological score (b) of DSS (black boxes), DSS + IEO-FMT (white boxes), DSS + CR-FMT (dotted boxes) and DSS + Envigo-FMT (striped boxes) treated mice. c Colonic expression of Tnf and II1b in DSS (black boxes), DSS + IEO-FMT (white boxes), DSS + CR-FMT (dotted boxes) and DSS + Envigo-FMT (striped boxes) treated mice d Comparison of relative abundancies of different taxa between Envigo- (outer chart), Charles River- (CR) (middle chart) or IEO-derived faecal microbiota. e Partial Least square-discrimination analysis (PLSD-DA) on metabolomics data between Envigo- (blue), Charles River- (CR, green) or IEO (red)-derived faecal microbiota. $\mathbf{f}$ Comparison of relative abundancies of different taxa between normobiotic-, IEO-, CR- Envigo- and dysbiotic-derived fecal microbiota. Statistical significance was calculated using a Mann-Whitney test for comparison within two groups or Kruskal-Wallis test with Dunn's multiple comparison correction within more than two groups. ${ }^{\star} P<0.05,{ }^{\star \star} P<0.01,{ }^{\star \star \star} P<0.001$ were regarded as statistically significant. Outliers were detected with Grubb's test. Non parametric distributions were represented as median +/- interquartile range. In Box and whiskers plots, centre line represents median; cross, represents mean; dots represent outliers. In (a-c) DSS $n=7$, DSS + IEO-FMT $n=10$, DSS + CR-FMT $n=10$, DSS + Envigo-FMT $n=10$. In (d, f) IEO-FMT $n=9$, CR-FMT $n=10$, Envigo-FMT $n=9, \mathrm{nFMT}=7, \mathrm{dFMT}=7$. In (e) DSS $n=5$, DSS + IEO-FMT $n=6$, DSS + CR-FMT $n=5$, DSS + Envigo-FMT $n=5$

microbial ecology, which were either completely absent or present in extremely low abundancies in normobiotic mice, were mostly Enterobacteriaceae, Bactaeriaceae, Rhodospirillaceae, Streptococcaceae, which have been shown to be increased both in colitic mice and IBD patients ${ }^{4,31}$ (Fig. 4f)

Taken together, these data indicate that beneficial effects of FMT are strictly dependent on the graft composition, and that only a healthy microbiota can ameliorate the intestinal inflammation.

FMT influences colonic immune cells relative abundance. Given the strict interdependence between intestinal immune system and the host microbiota ${ }^{45}$, we next aimed to explore immune pathways selectively modulated by FMT, We thus asked if therapeutic FMT administration in colitic mice induced variations in the frequencies and in the functional activities of the immune cell colonic infiltrate. The presence of specific bacterial strains in the gut has been linked to the differentiation and expansion of conventional ${ }^{11}$ and unconventional ${ }^{25} \mathrm{CD} 4^{+} \mathrm{T}$ cells. An increase of iNKT cell frequency, but not absolute numbers, (Fig. 5a) was observed in DSS-treated mice after FMT, while CD4 $+\mathrm{T}$ cells were decreased both in frequency and numbers (Fig. 5a), in line with their reduced proliferative capacity (Fig. 5b). Given that iNKT cells isolated from FMT-treated and untreated mice exhibited similar Ki67 expression (Fig. 5b), the increase of iNKT cell percentages on total $\mathrm{CD}^{+}$lymphocytes was likely a consequence of the overall reduction of $\mathrm{CD} 4^{+} \mathrm{T}$ cells rather than of their expansion (Fig. 5b). On the contrary, colonic CD8 ${ }^{+} \mathrm{T}$ cells frequency (Supplementary Fig. 6a) and phenotype (Fig. 5c) were not affected by FMT-treatment.

Interestingly, we found that FMT treatment was able to reduce the inflammation-driven expansion of the innate lymphocytes ILC2 and ILC $3^{46,47}$ in the lamina propria of acute DSS-treated mice (Supplementary Fig. 6b, c)

Antigen presenting cells (APC) and immune cells of myeloid origin infiltrating the inflamed lamina propria have been shown to contribute to sustain and propagate intestinal inflammation ${ }^{2}$. Following therapeutic FMT, a reduction in $\mathrm{F} 4 / 80^{+}$macrophages and $\mathrm{CD} 11 \mathrm{~b}^{+} \mathrm{Ly}_{6 \mathrm{G}}{ }^{+}$neutrophils frequency (Fig. 5d) and partially in absolute numbers (Fig. 5e) was observed. In contrast, $\mathrm{CD} 19^{+} \mathrm{B}$ cells and CD11c ${ }^{+}$dendritic cells were mostly unaffected (Fig. 5d, e).

Further, several evidences suggest that mucosal T-helper cells activation during intestinal inflammation depends on antigenic stimulation $^{2}$. After FMT, both the number of colonic MHC-IIexpressing professional APC, including dendritic cells and macrophages, (Fig. 5f) and MHC-II expression levels on APC (Fig. 5g) were strongly reduced, suggesting that FMT might directly act on the antigen presenting capacity of professional APC.
To note, similar findings were observed in the TNBS-induced experimental colitis model (Supplementary Figure 7)

Taken together, these data indicate that therapeutic FMT exerts a specific effect on infiltrating immune cells population frequency and phenotype.

$\mathrm{CD}^{+} \mathrm{T}$ cells cytokine skewing requires antigen presentation. We next evaluated if FMT administration might influence specific immune cells functional activities that are directly correlated to bacterial antigens presentation.

The faeces of untreated, DSS-treated and FMT-treated mice were collected and used to stimulate in-vitro intestinal lamina propria mononuclear cells (LPMC) freshly isolated from healthy mice (Fig. 6a).

As observed in vivo, MHC-II levels on dendritic cells, macrophages and monocytes were down-regulated upon in vitro exposure to normal (untreated) or FMT-derived microbiota (Fig. 6b). Similarly, the expression of CD86, a costimulatory molecule critically involved in T cell stimulation, was reduced on APC exposed to untreated or FMT-derived microbiota as compared to those exposed to the faeces of DSS-treated mice (Supplementary Fig. 8a).

Cytokines produced by mucosal antigen presenting cells play a pivotal role in the initiation and propagation of intestinal inflammation, as in the maintenance of homeostasis ${ }^{48}$. To evaluate the cytokine milieu generated upon exposure to FMTderived microbiota, we analysed the supernatants of stimulated intestinal LPMC (Fig. 6c). Interestingly, LPMC exposed to FMTderived microbiota showed reduced levels of pro-inflammatory cytokines, such as TNF, IL1 $\beta$ and IFN $\gamma$ (Fig. 6c), which were instead increased in the supernatants of LPMC exposed to DSSderived microbiota.

FMT-derived microbiota was also capable to differently skew the cytokine profile of antigen presenting cells (Fig. 6d, e). Indeed, the frequency of pro-inflammatory TNF-producing intestinal dendritic cells and macrophages was strongly reduced upon exposure to FMT-derived microbiota (Fig. 6d). Conversely, FMTderived microbiota increased the frequency of IL-10-producing intestinal dendritic cells and monocytes (Fig. 6e).

Correspondingly, $\mathrm{CD}^{+} \mathrm{T}$ cell expansion occurred only after stimulation with DSS-derived, but not with normal or FMTderived microbiota (Fig. 6f). To note, FMT-derived microbiota slightly increased the frequency of Foxp3 + Treg cells (Supplementary Fig. 8b).

DSS-derived microbiota also induced a strong upregulation of CD69 on intestinal $\mathrm{CD}^{+}{ }^{+} \mathrm{T}$ cells, while untreated or FMTderived microbiota failed to do so (Fig. 6g). Likewise, only FMTderived microbiota significantly increased IL-10 secretion by $\mathrm{CD} 4+\mathrm{T}$ cells, in an MHC-II dependent fashion (Fig. 6h). Importantly, also IFN $\gamma$ secretion was dependent on antigen presentation (Fig. 6h). 
a

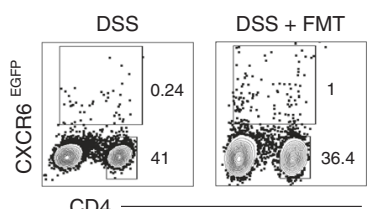

DSS
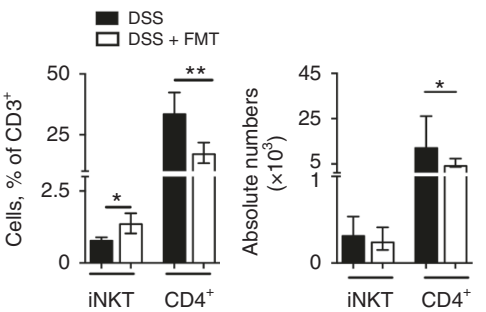

b
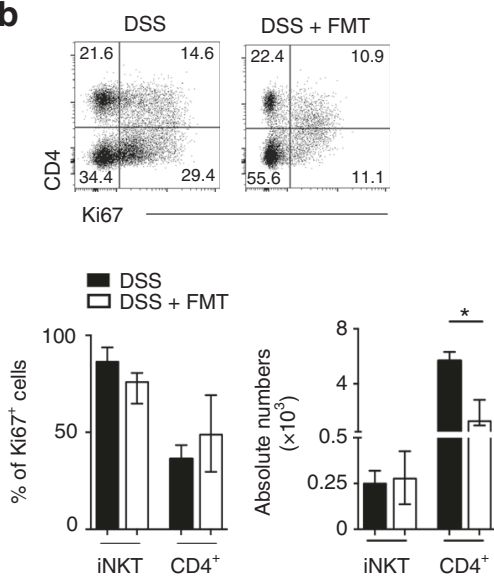

d
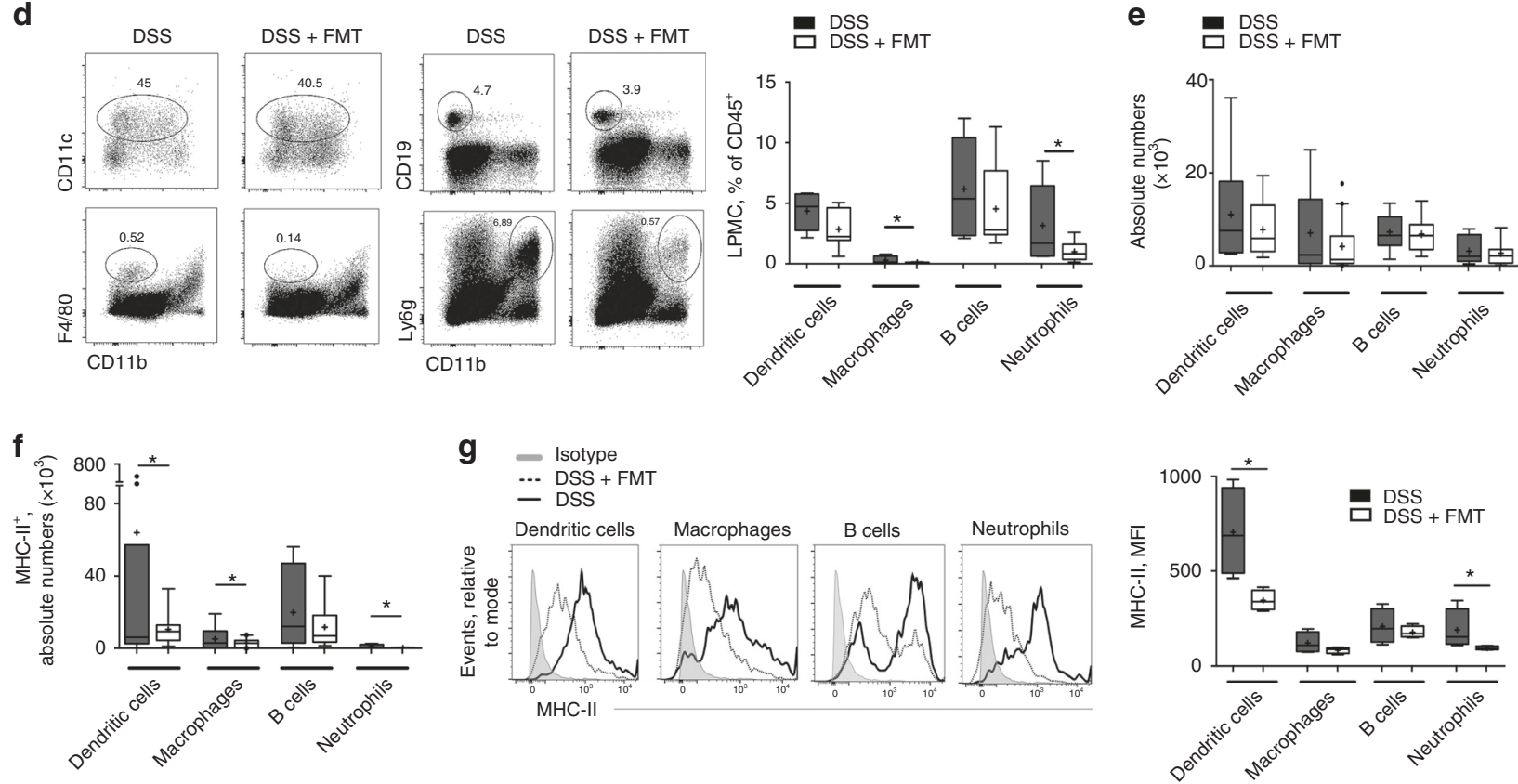

Fig. 5 Therapeutic FMT modulates immune cell frequencies and phenotypes. a Representative dot plots (upper panels), frequencies and absolute numbers (lower panels) of colonic CD4 ${ }^{+}$T cells and iNKT cells in DSS-treated (black bars) and FMT-treated (white bars) mice 11 days after starting DSS administration. b Representative dot plots, (upper panels), frequencies and absolute numbers (lower panels) of Ki67-expressing colonic CD4 ${ }^{+} \mathrm{T}$ cells and iNKT cells in DSS-treated (black bars) and FMT-treated (white bars) mice. c Representative dot plots, (upper panels), frequencies and absolute numbers (lower panels) of Ki67 and CD107a- expressing colonic CD8 ${ }^{+}$T cells in DSS-treated (black bars) and FMT-treated (white bars) mice. $\mathbf{d}$ Representative dot

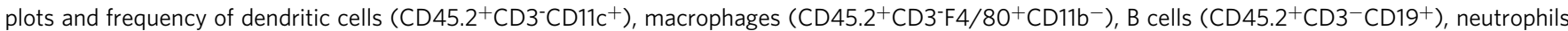
$\left(\mathrm{CD} 45.2^{+} \mathrm{CD}^{-}{ }^{-} \mathrm{Ly} 6 \mathrm{~g}^{+} \mathrm{CD}_{11} \mathrm{~b}^{+}\right)$in DSS-treated (left panels) and FMT-treated (right panels) mice 11 days after starting DSS administration. e Absolute numbers of colonic dendritic cells, macrophages, B cells, neutrophils in DSS-treated (black boxes) and DSS + FMT-treated (white boxes). f Absolute numbers of MHC-II expressing colonic dendritic cells, macrophages, B cells and neutrophils in DSS (white boxes) and DSS + FMT-treated (black boxes) mice. $\mathbf{g}$ Representative histograms of MHC-II expression and cumulative Mean fluorescence intensity (MFI) on colonic dendritic cells, macrophages, B cells, neutrophils in DSS (black boxes) and DSS + FMT (white boxes) treated mice. Statistical significance was calculated using a Mann-Whitney test for comparison within two groups or Kruskal-Wallis test with Dunn's multiple comparison correction within more than two groups. ${ }^{\star} P<0.05$, ${ }^{\star \star} P<0.01$, ${ }^{\star \star \star} P$ $<0.001$ were regarded as statistically significant. Outliers were detected with Grubb's test. Non parametric distributions were represented as median $+/-$ interquartile range. In Box and whiskers plots, centre line represents median; cross, represents mean; dots represent outliers. In (a-c) DSS $n=12$, DSS + FMT $n=12 . \ln (\mathbf{d}-\mathbf{g})$ DSS $n=16$, DSS + FMT $n=16$

Taken together these data confirm a crucial role for bacterial antigen presentation in the tolerogenic skewing of innate and adaptive colonic immune populations upon FMT treatment.

IL-10 critically contributes to FMT beneficial effects. In vitro experiments indicated that IL-10 production by intestinal immune cells might be critically involved in the tolerogenic mechanisms triggered by therapeutic FMT during experimental colitis. We thus evaluated if these mechanisms occurred in vivo upon FMT, and if IL-10 production by immune cells might be responsible for the observed therapeutic effects of FMT.

Higher amounts of colonic IL-10 (Fig. 7a) as well as increased frequencies of IL-10-producing APC (Fig. 7b, c) and CD4 ${ }^{+} \mathrm{T}$ and iNKT cells (Fig. 7d) were observed in the colons of FMT-treated mice as compared to DSS-treated mice. Interestingly, the increased IL-10 secretion by $\mathrm{T}$ cells observed in FMT-treated 
a
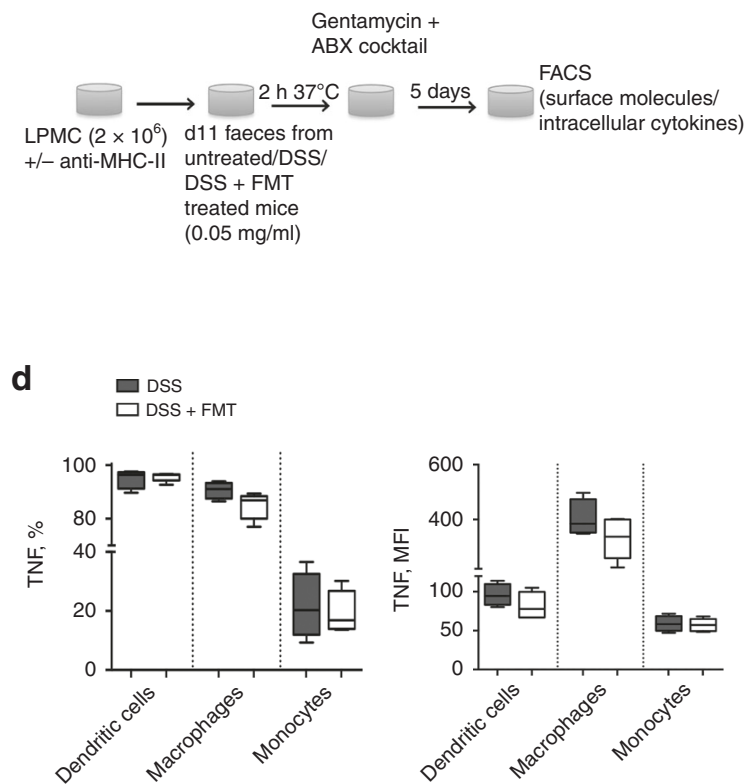

b

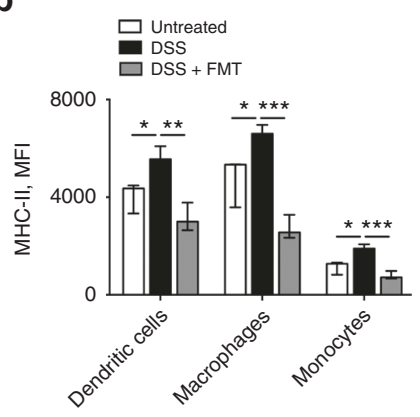

c

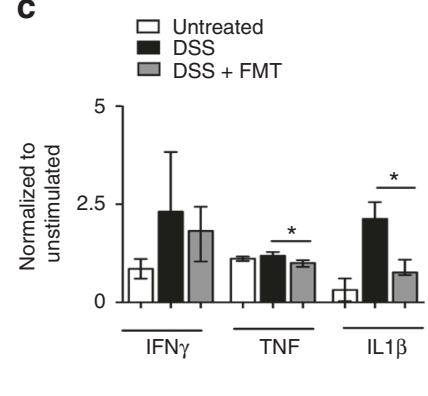

e

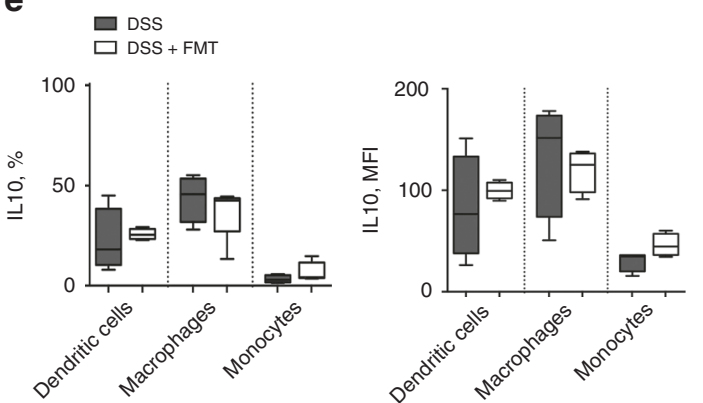

f

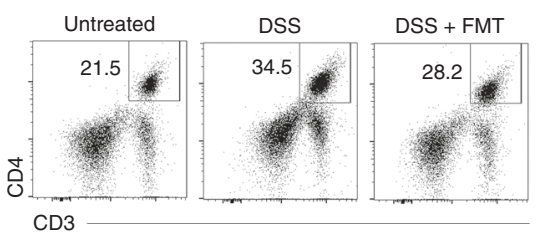

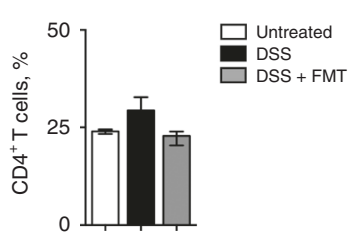

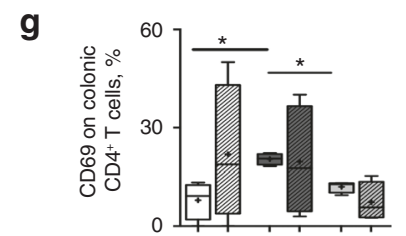

$-+-+-+$

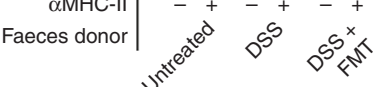

h
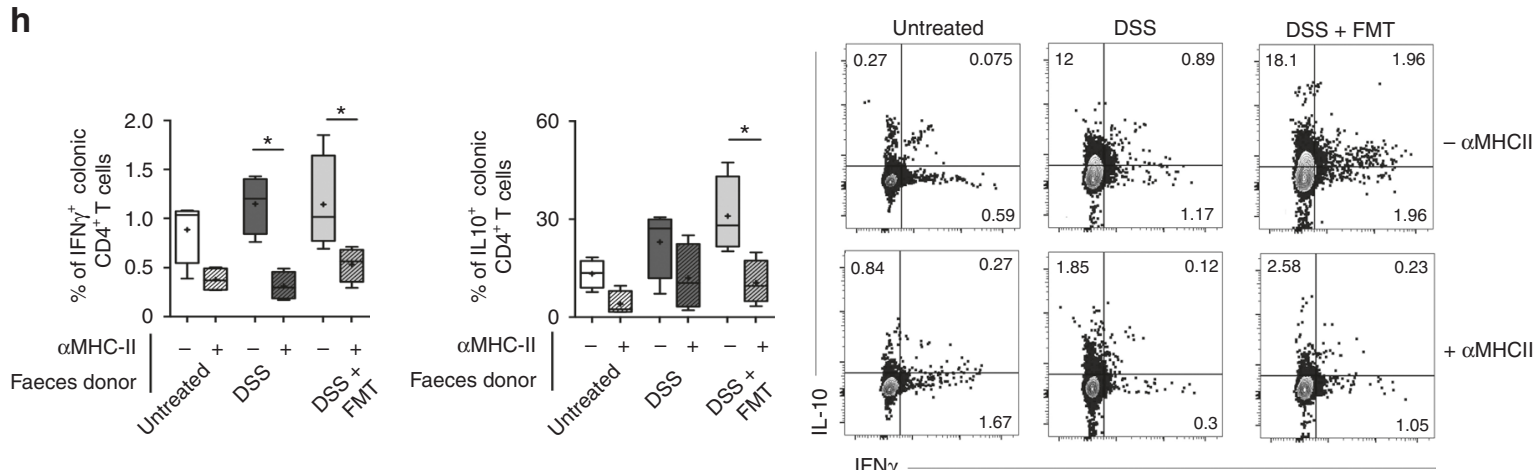

Fig. 6 Bacterial antigens presentation is required for $\mathrm{CD}^{+}{ }^{+} \mathrm{T}$ cells cytokine skewing. a Schematic representation of the experiment. b Mean fluorescence intensity (MFI) of MHC-II on dendritic cells, macrophages, monocytes, neutrophils exposed in vitro to faeces isolated from untreated (white bars), DSS (black bars) and DSS + FMT (grey bars) treated mice. c IFNg, TNF and IL1b cytokines levels in supernatants of LPMC in vitro exposed to faeces isolated from untreated (white bars), DSS (black bars) and DSS + FMT (grey bars) treated mice. Cytokines levels were normalised to unstimulated spontaneous secretion by LPMC. d, e Frequencies (left graphs) and MFI (right graphs) of TNF- (d) and IL-10- (e) secreting dendritic cells, macrophages and monocytes exposed in vitro to faeces isolated from DSS (dark gray bars) and DSS + FMT (white bars) treated mice (f) Representative dot plots and frequencies of $\mathrm{CD}^{+}{ }^{+} \mathrm{T}$ cells exposed in vitro to faeces isolated from untreated (white bars), DSS (black bars) and DSS + FMT (grey bars) treated mice. $\mathbf{g}$ CD69 expression on intestinal CD4 ${ }^{+}$T cells exposed in vitro to faeces isolated from untreated (white boxes), DSS (black boxes) and DSS + FMT (grey boxes) treated mice in the presence (striped bars) or absence (filled bars) of blocking $\alpha$ MHC-II antibodies. $\mathbf{h}$ Frequencies and representative dot plots of IFN $\gamma$ and $\mathrm{IL}-10$ secreting intestinal CD4 ${ }^{+}$T cells exposed in vitro to faeces isolated from untreated (white bars), DSS (black bars) and DSS + FMT (grey bars) treated mice in the presence (striped bars) or absence (filled bars) of blocking $\alpha \mathrm{MHC}-\mathrm{Il}$ antibodies. Statistical significance was calculated using a Mann-Whitney test for comparison within two groups or Kruskal-Wallis test with Dunn's multiple comparison correction within more than two groups. ${ }^{\star} P<0.05$, ${ }^{\star \star} P<$ $0.01,{ }^{\star \star \star} P<0.001$ were regarded as statistically significant. Outliers were detected with Grubb's test. Non parametric distributions were represented as median + /- interquartile range. In Box and whiskers plots, centre line represents median; cross, represents mean; dots represent outliers. In all panels UT $n=5$, DSS $n=7$, DSS + FMT $n=9$ 
a

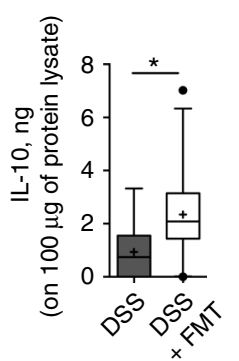

b $\quad$ - Isotype - DSS

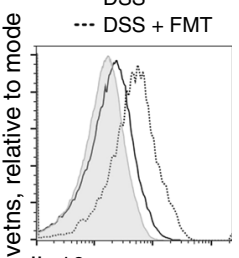

C

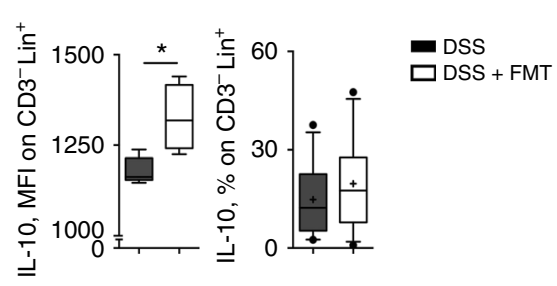

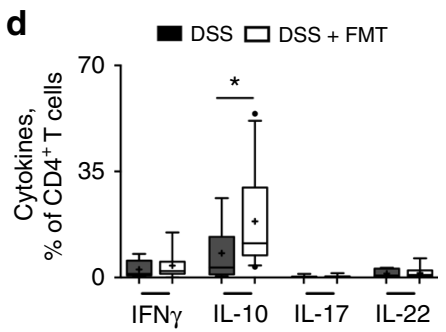

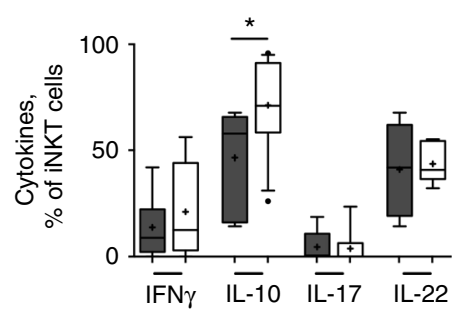

e
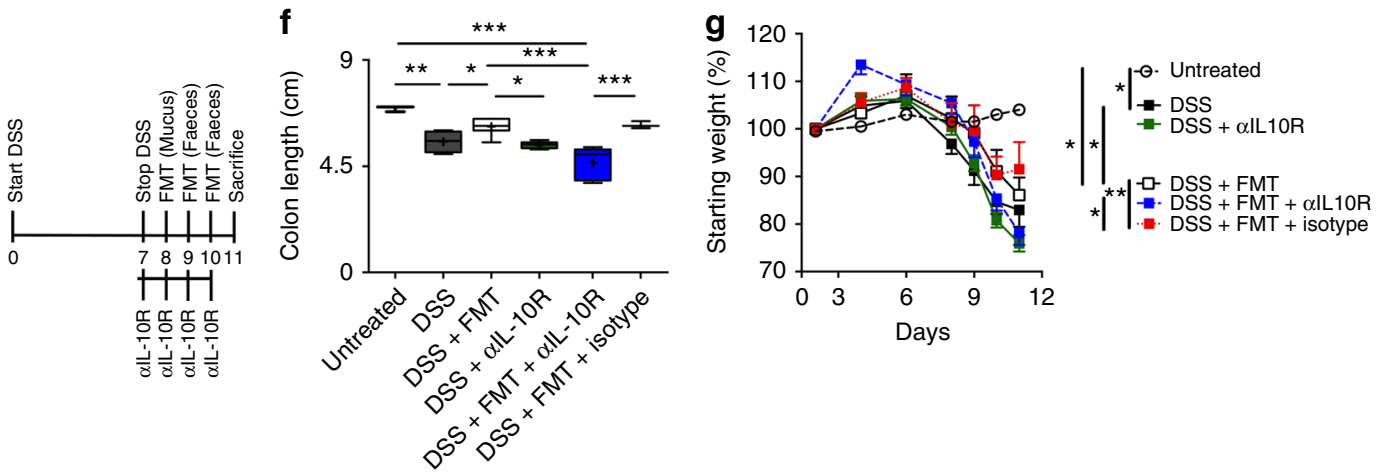

h
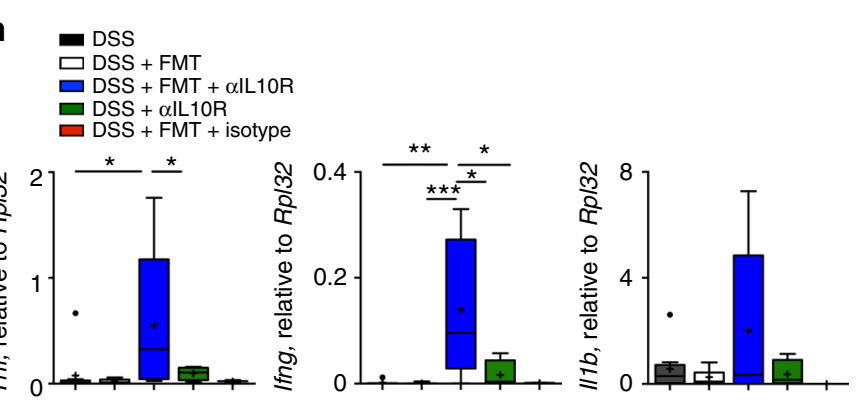

i

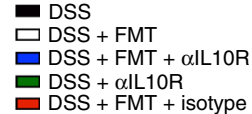

$\square \mathrm{DSS}+\alpha \mathrm{LL} 10 \mathrm{R}$
$\square \mathrm{DSS}+\mathrm{FMT}+$ isotype

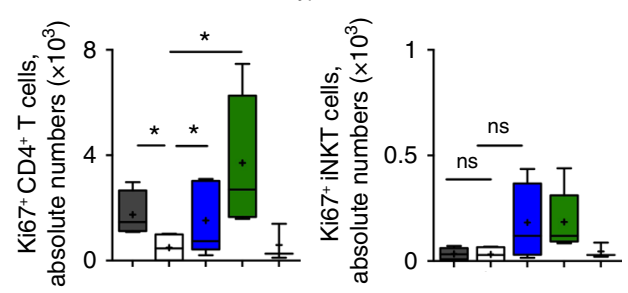

Fig. 7 IL-10 contributes to FMT beneficial therapeutic effects. a IL-10 colonic expression in DSS-(black boxes) and DSS + FMT-(white boxes) treated mice. b, c Representative histograms of IL-10 expression (b) and cumulative Mean fluorescence intensity (MFI) (c), left panel) and frequency (c, right panel) of IL10-producing colonic professional APC (CD45 $\left.{ }^{+} \mathrm{CD} 3^{-} \mathrm{MHC}-\mathrm{II}^{+}\right)$in DSS (black boxes) and DSS + FMT (white boxes) treated mice. $\mathbf{d}$ Summary of cytokines secreted by colonic CD4 ${ }^{+}$T cells and iNKT cells in DSS-treated (black boxes) and FMT-treated (white boxes) mice 11 days after starting DSS administration. e Schematic representation of the experiment. $\mathbf{f}, \mathbf{g}$ Colon length (f) and weight loss ( $\mathbf{g}$ ) of untreated mice (white dotted boxes/open circles), or receiving DSS (black boxes/black symbols), or DSS + FMT (white boxes/white square symbols) or DSS $+\alpha \mathrm{LL}-10 \mathrm{R}$ (green boxes/green symbols), or DSS + FMT $+\alpha$ IL-10R (blue boxes and blue symbols) or mice receiving DSS + FMT $+\alpha$ IL-10R isotype antibody (red boxes/red symbols). h Colonic expression of Tnf, Ifn $\gamma$ and IL1 $\beta$ in DSS (black boxes), DSS + FMT (white boxes), DSS $+\alpha \mid \mathrm{LL}-10 \mathrm{R}$ (blue boxes), DSS + FMT $+\alpha I L-10 R-$ (green boxes) and DSS + FMT $+\alpha$ IL-10R-isotype-antibody-treated mice (red boxes). i Absolute numbers of KI67 expressing colonic CD4+ $\mathrm{T}$ cells (left panels) and iNKT cells (right panels) in DSS (black boxes), DSS + FMT (white boxes), DSS $+\alpha \mid \mathrm{L}-10 R$ (blue boxes), DSS + FMT $+\alpha \mid \mathrm{L}-10 \mathrm{R}$ (green boxes) and DSS $+\mathrm{FMT}+\alpha \mathrm{IL}-10 \mathrm{R}$ isotype antibody (red boxes). Statistical significance was calculated using a Mann-Whitney test for comparison within two groups or Kruskal-Wallis test with Dunn's multiple comparison correction within more than two groups. ${ }^{\star} P<0.05,{ }^{\star \star} P<0.01,{ }^{\star \star \star} P<0.001$ were regarded as statistically significant. Outliers were detected with Grubb's test. Non parametric distributions were represented as median $+/-$ interquartile range. In Box and whiskers plots, centre line represents median; cross, represents mean; dots represent outliers. In (a) DSS $n=15, \mathrm{DSS}+\mathrm{FMT} n=26$. In $(\mathbf{b}-\mathbf{d})$ DSS $n=$ $14, \mathrm{DSS}+\mathrm{FMT} n=17 . \mathrm{In}(\mathbf{e}-\mathbf{i}) \mathrm{DSS}, \mathrm{DSS}+\mathrm{FMT} n=4 ; \mathrm{DSS}+\alpha \mathrm{IL}-10 \mathrm{R}, \mathrm{DSS}+\mathrm{FMT}+\alpha \mathrm{LL}-10 \mathrm{R} n=5, \mathrm{DSS}+\mathrm{FMT}+$ isotype $n=3$ 
mice normalised upon inflammation resolution (Supplementary Fig. 9b). To test the contribution of IL-10 on the antiinflammatory properties of FMT, DSS-treated mice were administered FMT concomitantly to IL-10 receptor (IL-10R) blockade (Fig. 7e). Inhibition of the tolerogenic functions of IL-10 on IL-10R expressing cells, such as antigen presenting cells (APC), $\mathrm{T}$ cells and epithelial cells ${ }^{49}$, hampered FMT protective effects as shown by reduced colon length (Fig. 7f), increased weight loss (Fig. $7 \mathrm{~g}$ ) and higher colonic expression of Tnf, Ifny and $\operatorname{Il} 1 \beta$ (Fig. $7 \mathrm{~h}$ ). These effects were not observed when IL-10R was blocked in colitic mice without a concomitant FMT administration, suggesting a direct contribution of the microflora in the IL-10-mediated control of inflammation. As expected, IL10R blockade reverted the inhibition of $\mathrm{CD} 4^{+} \mathrm{T}$ cells proliferation occurring upon FMT (Fig. 7i). IL-10R blockade also affected IL-10 production by $\mathrm{T}$ and iNKT cells, possibly through a feedback regulatory loop (Supplementary Fig 9c).

Protective bacteria selective elimination impairs FMT effect. To further correlate the gut microbiota composition to the induction of protective immune-mediated functions during intestinal inflammation, therapeutic FMT was performed with mucus and faeces isolated from mice previously pre-treated for two weeks with different antibiotics targeting either Grampositive organisms (vancomycin), Gram- negative bacteria (streptomycin), strict anaerobes (metronidazole) or having a broad spectrum bacterial depletion capability (ABX) (Fig. 8a) ${ }^{50}$.

The taxonomic composition of the different donor microbiota was analysed by $16 \mathrm{~S}$ rRNA gene sequencing before administration to colitic mice. As expected, the a-diversity in antibiotictreated samples was lower as compared to those derived from normobiotic samples (Fig. 8b), evidence confirmed also by plating of the faecal material in aerobic and anaerobic conditions (Fig. 8c).

The detailed phylogenetic analysis of the taxonomic composition highlighted a relevant dysbiosis in the antibiotic- treated donor samples as compared to the untreated ones (Fig. 8d, e). In particular, metronidazole treatment favored the selective persistence of Lactobacillaceae, Bifidobacteriaceae and Erysipelotrichaceae, as well as of Ruminococcacceae and Bacteroidales S24-7, families belonging to the protective normobiotic microbial ecologies $^{31}$ previously described in Fig. 4. On the contrary, pathobionts such as Christensenellaceae, Burkholderiales, Listeriaceae and Gastranaerophilales significantly emerged in Vancomycin-treated samples at the expenses of the abovementioned protective families. Streptomycin-treatment, instead, favoured a limited presence of Erysipelotrichaceae and Ruminococcaceae while not expanding pathobionts, but rather allowing the survival of families whose function could be protective or detrimental according to the context ${ }^{1,51}$. As a proof of concept, the microbial population of broad spectrum antibiotic-treated mice was completely depleted, as confirmed by the plating of their faecal material (Fig. 8c).

Of note, FMT performed with metronidazole-treated microbiota retained a full capability to control intestinal inflammation (Fig. 8f, g and Supplementary Fig 10). Importantly, this was associated with a selective increased production of IL-10 by colonic APC (Fig. 8h), as to confirm the previously suggested IL10 promoting activity of Lactobacillaceae and Bifidobacteriaceae ${ }^{52}$.

In conclusion, our data show that the gut microbiome modifications after FMT exert a profound impact on the mucosal immune system. The composition of the microbial ecology transferred by FMT is pivotal to its beneficial anti-inflammatory effects by supporting changes in immune cell frequencies, the reduction of colonic if $n \gamma$ and $i l 1 \beta$, the increase in antimicrobial peptides and mucins, and the decrease of bacterial antigen presentation by APC. Most importantly, normobiotic FMT induces the skewing of innate and adaptive immune cells toward a tolerogenic IL-10 secreting cytokine profile that, altogether, concur to restore intestinal homeostasis (Fig. 9).

\section{Discussion}

FMT is becoming the first-line therapy in antibiotic resistant recurrent $\mathrm{CDI}^{14,15}$. However, its therapeutic application to other gastrointestinal diseases is at the very beginning and data on its mechanism of action during intestinal inflammation are still scarce. While it is known that restoration of normobiosis correlates with clinical remission in successful trials involving UC patients ${ }^{16-18}$, it is still unclear whether FMT might have an effect on the immune system. To date, data in $\mathrm{BALB} / \mathrm{c}$ mice suggest that FMT can induce $\mathrm{CD} 4{ }^{+} \mathrm{CD} 25^{+}$regulatory $\mathrm{T}$ cells and reduce colonic expression of Ill $\beta$ and If $\gamma^{19}$.

In our study, we show for the first time that the manipulation of the gut microbiota by FMT induces variations in both innate and adaptive immune cell frequencies and cytokine profiles and that this correlates with a general amelioration of the inflammatory status in colitic animals. Moreover, analyses of the gut microbiota in our model showed that 3 days of therapeutic FMT are sufficient to introduce modifications in the dysbiotic microbiota, with a relevant change in the relative proportions of Firmicutes. These results are in agreement with findings from successful FMT clinical trials on UC patients and from animal models of intestinal inflammation. In particular, data in UC patients showed that clinical remission correlated with stable modifications of the gut microbiota towards functional normobiosis restoration ${ }^{16-18}$ and that this shift in intestinal microbial ecology was sufficient to trigger reduction of inflammatory genes such as if $n \gamma$, ill $\beta$ and $t n f^{53}$. Further, in IBD patients and in animal models of intestinal inflammation ${ }^{4,33,54}$ changes in the levels of Erysipelotrichaceae and Lactobacillacee have been described. Interestingly, we found that these taxa were also similarly altered in DSS-treated mice, and their abundances were restored upon therapeutically successful FMT.

It is now acknowledged that the gut microbiota composition is heavily influenced by age, gender, genotype, diet and environmental factors ${ }^{55}$. In this context, animal models are valuable tools to study complex biological phenomena, such as those occurring during intestinal inflammatory processes, while controlling confounding variables. It also emerged from several recent studies that different research institutions or commercial vendors might harbour variations in the microbiological environment ${ }^{39,40}$, leading to differences in microbiota composition. Here we showed that the microbiota derived from three different sources were equally capable to control intestinal inflammation in our experimental model, and this capability was dependent on the presence of a core microbial ecology composed by Bacteroidales S24-7, Lachnospiraceae, Lactobacillaceae, Ruminococcaceae, Rikenellaceae, Bifidobacteriaceae and Erysipelotrichaceae. As recently shown also in metabolic diseases ${ }^{41}$ and in murine models of intestinal inflammation ${ }^{31}$, this core ecology consists of bacteria from different phyla sharing similar metabolic functions (i.e: SCFA production, $\mathrm{Ph}$ control, free radicals scavenging) and, when maintained at a certain population level, capable to create environmental conditions sufficient to inhibit the growth of pathogenic species/ pathobionts and support optimal host health.

A healthy gut environment, shaped by the presence of a healthy functional microbial ecosystem, is thus fundamental to instruct the immune system towards homeostasis. 
a

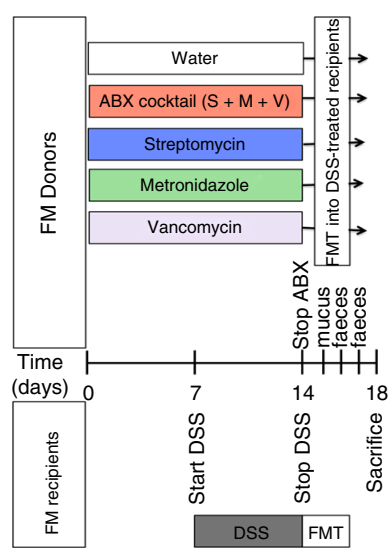

b

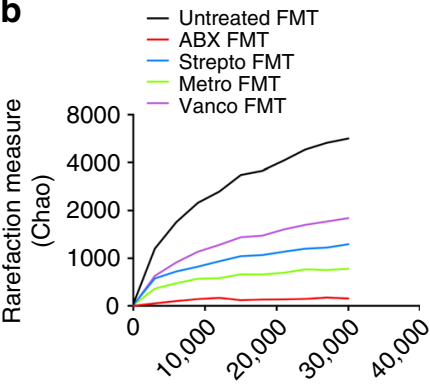

Number of sequences

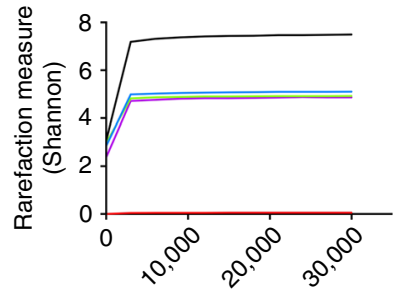

Number of sequences d

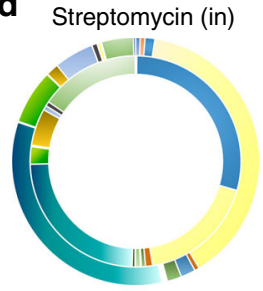

Bifidobacteriaceae - Coriobacteriaceae

Bacteroidales S24-7 group

- Porphyromonadaceae

- Prevotellacea

Rikenellaceae

- Clostridiales vadin BB60 group

Lachnospiraceae

Ruminococcaceae

Erysipelotrichacea

Lactobacillacea

Rhodispirillacea

Verrucomicrobiaceae

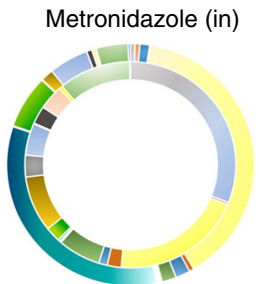

Bifidobacteriaceae

Coriobacteriaceae

Bacteroidaceae

Bacteroidales S24-7 group

- Porphyromonadaceae

- Prevotellaceae

Lachnospiraceae

- Ruminococcaceae

- Erysipelotrichaceae

- Enterococcaceae

- Lactobacillaceae

- Alcaligenaceae

Enterobacteriaceae

Rhodispirillaceae

Verrucomicrobiaceae

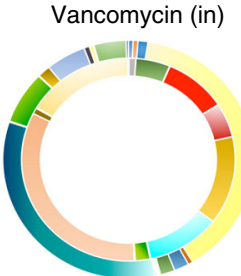

Bifidobacteriaceae

Propionibacteriacea

Bacteroidaceae

Bacteroidales S24-7 group

Porphyromona

Prevotellaceae

Gastranaerophilales order spp.

Listeriaceae

Christensenellaceae

Defluvitaleaceae

Ruminococcacea

Erysipelotrichaceae

Streptococcaceae

Veillonellaceae

- Aurkholderiales order spp Rhodispirillaceae
Verrucomicrobiaceae

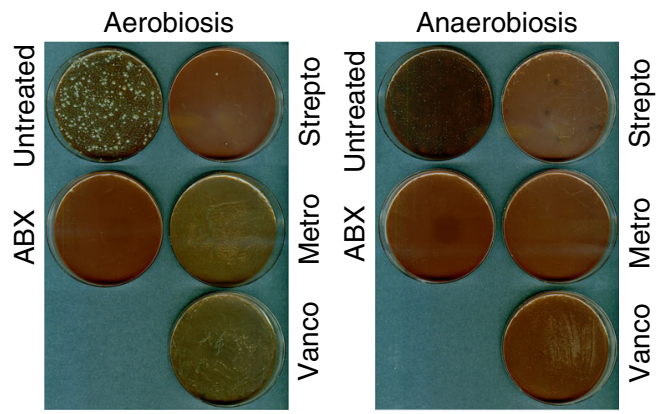

C f

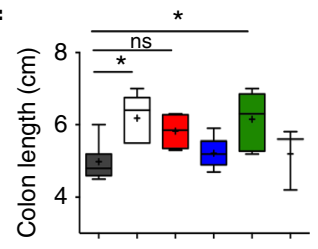

g

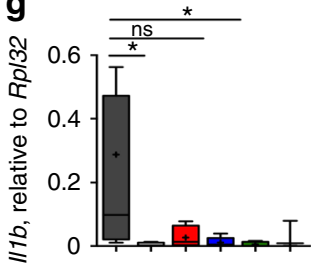

h

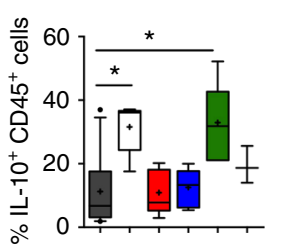

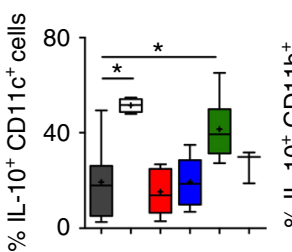
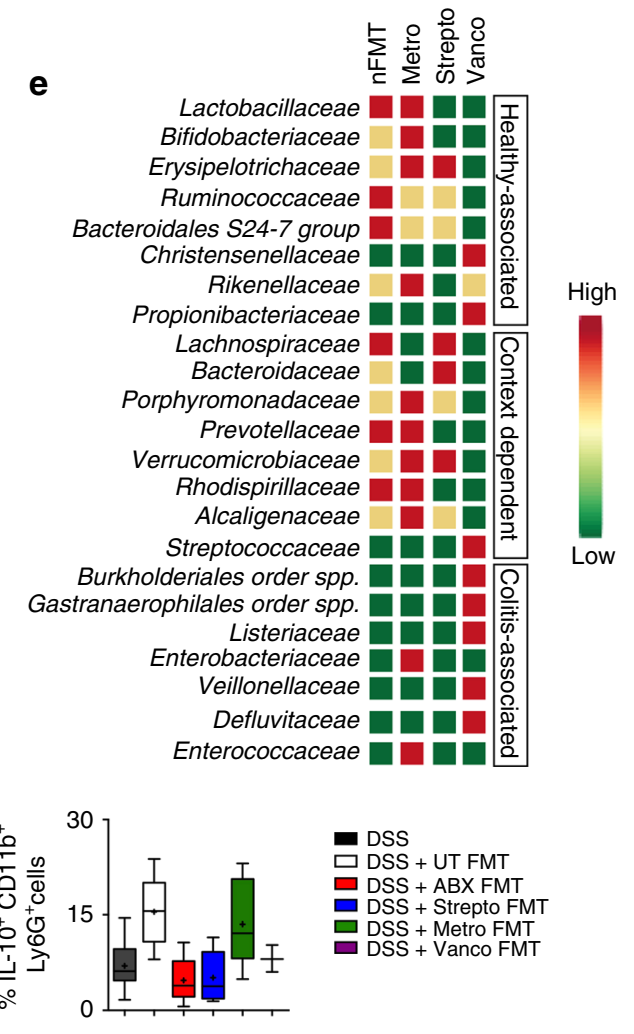

$\square$ DSS + UT FMT

DSS + ABX FMT

DSS + Metro FMT

DSS + Vanco FMT

Several reports describe the pivotal role of $\mathrm{T}$ cells as key players in the initiation and maintenance of intestinal inflammation. For instance, we $\mathrm{e}^{56}$ and others ${ }^{57,58}$ reported that IBD patients show increased amounts of intestinal Th1, Th17 and Th1/17 cells whose pathogenic role has been associated to the recognition of bacterial antigens ${ }^{7,59,60}$. In addition to conventional $\mathrm{CD} 4^{+}$
T cells, other T cell subsets, like iNKT cells, can secrete IL17A and IFN $\gamma$ in the gut and affect bacterial colonization of the intestine ${ }^{20,61}$ but their role in intestinal inflammation is less well defined $^{62}$.

In our study, in vitro stimulation of intestinal lamina propria cells with faecal content from untreated, DSS or FMT-treated 
Fig. 8 Antibiotic treatment selectively abolishes FMT beneficial effects. a Schematic representation of the experiment. b Rarefaction curves showing microbial richness (Chao1 index,top panel) and microbial richness and evenness (Shannon index,bottom panel). Black line, untreated-, red line ABXFMT-, blue line Strepto FMT-, Green line Metro FMT-, Violet line Vanco FMT- derived samples. c Plating of faecal material derived from untreated, treated with the antibiotic cocktail (ABX) or with streptomycin (Strepto), Metronidazole (Metro) or Vancomycin (Vanco) in aerobiosis (left) and anaerobiosis (right). d Comparison of relative abundancies of different taxa between faecal microbiota obtained from normobiotic (outer chart) and Streptomycin-treated mice (inner chart, left panel), or Metronidazole-treated (inner chart, middle panel) or Vancomycin-treated mice(inner chart, right panel). e Heat map comparing the expression levels of the different taxa between faecal microbiota obtained from normobiotic, Streptomycin-, Metronidazole- or Vancomycin-treated mice. f, $\mathbf{g}$ Colon length (f) and illb colonic expression (g) in DSS-treated (black boxes), DSS + Untreated FMT (white boxes), DSS + ABX FMT (red boxes), DSS + Streptomycin FMT (blue boxes), DSS + Metronidazole FMT (green boxes) and Vancomycin (Violet boxes)-treated mice. $\mathbf{h}$ Frequencies IL-10 secreting colonic total CD45 + immune cell populations or gated dendritic cells (CD11c + ), neutrophils (Cd11b + Ly6g + ), Macrophages $(C d 11 b+F 4 / 80$ + ), B cells $(C D 19+)$ and CD4 ${ }^{+}$T cells isolated from DSS-treated (black boxes), DSS + untreated FMT (white boxes), DSS + ABX FMT (red boxes), DSS + Streptomycin FMT (blue boxes), DSS + Metronidazole FMT (green boxes) and DSS + Vancomycin FMT (Violet boxes)-treated mice. Statistical significance was calculated using a Mann-Whitney test for comparison within two groups or Kruskal-Wallis test with Dunn's multiple comparison correction within more than two groups. ${ }^{\star} P<0.05,{ }^{\star \star} P<0.01,{ }^{\star \star \star} P<0.001$ were regarded as statistically significant. Outliers were detected with Grubb's test. Non parametric distributions were represented as median+/- interquartile range. In Box and whiskers plots, centre line represents median; cross, represents mean; dots represent outliers. In (b-e) UT $n=4$; DSS, DSS + Abx FMT $n=5$; DSS + Strepto FMT, DSS + Metro FMT $n=6$; DSS + FMT Vanco $n=3$. $\ln (\mathbf{f}-\mathbf{h})$ UT $n=4$; DSS $n=5$; DSS + Abx FMT, DSS + Strepto FMT, DSS + Metro FMT, DSS + FMT Vanco $n=6$

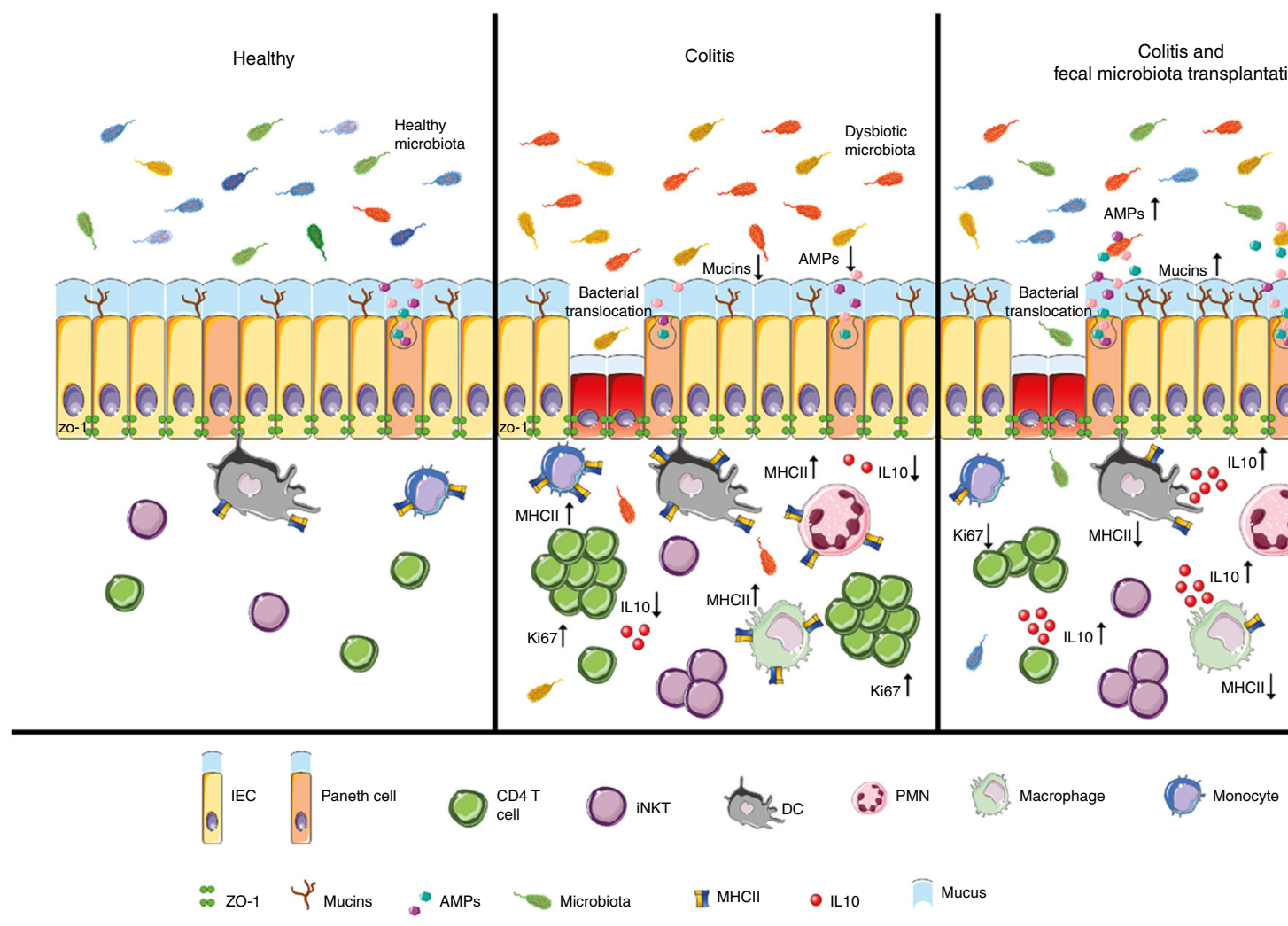

Fig. 9 Graphical abstract of events occurring in the intestinal mucosa in healthy conditions, during intestinal inflammation caused by DSS administration and during faecal transplantation in colitic mice. Adapted from SERVIER MEDICAL ART (CC BY 3.0 Licence)

mice induced a different cytokine profile in both innate and adaptive immune cells, suggesting that the alterations in the microbiota ecology during colitis and upon FMT are directly linked to changes in the functional status of the mucosal immune system.

Additionally, our data indicate that variations in the intestinal microbial ecology are also capable to skew, both in vitro and in vivo, the cytokine profile of antigen presenting cells from proinflammatory to tolerogenic ${ }^{45}$.

In particular, we show that in acute models of intestinal inflammation, IL-10 secretion by APC and T cells upon FMT is temporally linked to the resolution of inflammation, confirming a direct contribution of IL-10-mediated functional activities in intestinal homeostasis ${ }^{63}$ also in the context of FMT. IL-10 is an anti-inflammatory cytokine known to critically maintain intestinal immune homeostasis ${ }^{64}$. IL-10-deficient mice and patients with genetic defects in the IL-10/IL-10R pathway develop intestinal inflammation in the presence of a normal gut microflora. Moreover, the presence of specific commensal bacteria such as Lactobacilli and Bifidobacteria have been directly associated to the secretion of tolerogenic IL- $10^{52}$ and has been implicated in the maintenance of intestinal homeostasis $^{65}$. Several studies report that a probiotic preparation (\#VSL3) composed by a cocktail of eight strains of Lactobacilli and Bifidobacteria is effective in controlling intestinal inflammation ${ }^{32}$ 
The resolution of intestinal inflammation has been also linked to the activation and the functional plasticity of $\mathrm{CD}^{+} \mathrm{T}$ cells from pro-inflammatory to tolerogenic/IL-10-producing subtypes $^{63,66}$. Upon therapeutic FMT, both CD4+ and iNKT cells produce IL-10. Importantly, since IL-10 also negatively affects the proliferative capacity of cells the overall increased availability of colonic IL-10 might be directly responsible for the reduced proliferative capacity of $\mathrm{T}$ cells together with the decreased antigen presentation by APC, as shown by their reduced levels of MHCII.

In this manuscript we show that colonic APC, such as neutrophils and macrophages, both recruited in the inflamed gut ${ }^{67,68}$ are also strongly reduced in frequency and absolute number upon therapeutic FMT. This is in line with findings showing that macrophages contribute to IBD pathogenesis and are direct target of anti-TNF therapy, which induces their apoptosis ${ }^{69}$ and/or a regulatory $\mathrm{M} 2$ phenotype ${ }^{67}$.

Finally, we also show that the levels of MHCII and the frequency of $\mathrm{MHCII}^{+}$professional APC in the colonic lamina propria are strongly down-regulated upon FMT. Importantly, colonic HLA-DR expression levels discriminate between healthy, quiescent and active IBD patients ${ }^{70}$, confirming a prominent role of MHCII-dependent antigen presentation in IBD immunopathology. Since IFN $\gamma$ up-regulates MHCII molecules, the observed reduction of colonic IFN $\gamma$ after FMT could explain the decrease of MHCII surface levels and it might be instrumental for reducing the presentation of bacterial antigens to $\mathrm{CD} 4^{+} \mathrm{T}$ cells.

In conclusion, we demonstrate that modulation of intestinal microbiota by FMT during experimental colitis exerts multiple effects in both adaptive and innate mucosal immune responses. The restoration of normobiosis, possibly also through cooperative interactions among commensal species, could be the first hint to simultaneously trigger several immune pathways leading to tolerogenic functions of innate and adaptive immune cells that altogether contribute to the resolution of the inflammatory processes. Further studies will highlight if defined microbial species or community-based effects contribute to tolerogenic mechanisms in the gut.

These findings on overall represent an important contribution toward the elucidation of the complex interplay between the immune system and the gut microbial ecosystem and are instrumental for better understanding the immune events occurring during therapeutic FMT in humans.

\footnotetext{
Methods

Mice. C57BL/6 mice (Charles River, IT) and CXCR6 EGFP/ + mice (B6.129P2Cxcr6tm1Litt/J; IMSR_JAX: 005693) background C57BL/6, purchased as GFP/GFP from JAX, USA, and bred to heterozigosity with C57BL/6 mice) of 8-10 weeks of age were housed at the IEO animal facility in SPF conditions. Experimental groups of mice receiving the different FMT treatments were kept in separated cages. Littermates of same sex and age were randomly assigned to the different experimental groups.

Animal procedures were approved by the Italian Ministry of Health (Auth. 127/ $15,27 / 13,913 / 16,415 / 17)$ and by the OPBA of the European Institute of Oncology, IEO, Italy.

Experimental colitis models. For the induction of DSS-induced acute colitis, mice were given $2 \%(\mathrm{w} / \mathrm{v})$ dextran sodium sulphate (DSS, MW $40 \mathrm{kD}$; TdB Consultancy) in their drinking water for 7 days followed by 2 days of recovery. The weight curve was determined by weighing mice daily. At sacrifice, colons were collected, their length was measured and divided in portions to be fixed in $10 \%$ formalin for histological analyses, snap-frozen for RNA extraction and for lamina propria mononuclear cells (LPMC) immunophenotyping.
}

Faecal microbiota transplantation (FMT). FMT was performed through ora gavage of mucus (first day) and faeces (second and third days) preparations from donor mice. This protocol facilitates the engraftment of the mucus-associated bacteria. Donor mice were untreated (normobiotic) donors, DSS-treated (dysbiotic) or treated with metronidazole $(1 \mathrm{~g} / \mathrm{L})$, vancomycin $(1 \mathrm{~g} / \mathrm{L})$, streptomycin $(2 \mathrm{~g} / \mathrm{L})$ or a cocktail of the three antibiotics combined in order to deplete distinct taxa of bacteria. Mucus was scraped from colons, diluted in PBS and administered to recipients at 1:1 ratio. Faeces were collected, diluted in PBS $(50 \mathrm{mg} / \mathrm{ml})$ and administered to recipients by oral gavage $(10 \mathrm{mg} / \mathrm{mouse})$ one day after the end of acute DSS administration.

For in vivo IL-10R blockade, mice were injected intraperitoneally with $250 \mu \mathrm{g}$ InVivoMAb anti-mouse IL-10R (BioXCell, clone 1B1.3 A), or its Isotype (BioXCell), daily for 4 days starting from one day before FMT treatment.

Murine cell isolation. For LPMC isolation, Peyer's Patches were removed, colonic lamina propria lymphocytes (LPL) were isolated via incubation with $5 \mathrm{mM}$ EDTA at $37^{\circ} \mathrm{C}$ for $30 \mathrm{~min}$, followed by further digestion with collagenase IV and DNase at $37^{\circ} \mathrm{C}$ for $1 \mathrm{~h}$. Cells were then separated with a Percoll gradient.

In some experiments after isolation cells were re-stimulated in vitro for $3 \mathrm{~h}$ with PMA/Ionomycin in the presence of Brefeldin A for cytokine secretion.

Flow cytometry analysis. Cells were stained with combinations of directly conjugated antibodies described in Supplementary Table 3. iNKT cells were identified by CXCR6-EGFP expression or mCD1d:PBS57 Tetramer (NIH Tetramer core facility) staining.

Intracellular staining of cytokines was performed after cells fixtion and permeabilization with Cytofix/Cytoperm (BD) before addition of the antibodies. Samples were analysed by a FACSCanto II flow cytometer (BD), gated to exclude nonviable cells. Data were analysed using FlowJo software (BD). The complete list of the antibodies and dyes used in the study are described in Supplementary Table 3.

RT-qPCR of tissue mRNA. Total RNA from colonic tissues was isolated using TRIZOL and Quick-RNA MiniPrep (ZymoResearch) according to manufacturer's specifications and following the MetaHIT project guidelines. cDNAs were generated from $1 \mu \mathrm{g}$ of total RNA with reverse transcription kit (Promega). Gene expression levels were evaluated by qPCR and normalized to Rpl32 gene expression. The primer sequences are collected in Supplementary Table 2.

Immunofluorescence. Intestinal samples were fixed overnight in paraformalde hyde, L-Lysine pH 7.4 and NaIO4 (PLP buffer). They were then washed, dehydrated in $20 \%$ sucrose for at least $4 \mathrm{~h}$ and included in OCT (Sakura). 10 $\mu$ m-thick sections were re-hydrated with $0.1 \mathrm{M}$ Tris $\mathrm{HCl} \mathrm{pH:} 7.4$ buffer and blocked with $0.3 \%$ triton X-100, $2 \%$ FBS $0.1 \mathrm{M}$ Tris- $\mathrm{HCl}$ buffer. Slides were incubated with the primary antibody (anti ZO-1 FITC, 1:100) for 2 hrs. Nuclei were counterstained with DAPI (1:30.000; Roche) and mounted with Vectashield (Vectorlabs).

Histological analysis. Tissue processing was performed with a LEICA PELORIS processor before paraffin embedding ${ }^{71}$. Murine samples were included using an automated system (SAKURA Tissue-Tek). After Hematoxylin and Eosin staining, snapshots of histology were taken using an Aperio CS2 microscope with a scanning resolution of 50,000 pixels per inch $(0.5 \mu \mathrm{m}$ per pixel with $10 \mathrm{x}$ objective and $2.5 \mu \mathrm{m}$ per pixel when scanning at $\times 4$ ). Scoring of disease activity was performed according to the criteria described in Supplementary Table 1.

Microbiota identification. Faeces and mucus scraped from the colon were stored at $-80^{\circ} \mathrm{C}$ until the DNA was extracted with G NOME DNA isolation kit (MP) following the protocol described in ${ }^{72}$ Partial $16 \mathrm{~S}$ rRNA gene sequences were amplified using primer pair Probio_Uni and /Probio_Rev, targeting the V3 region of the $16 \mathrm{~S}$ rRNA gene sequence ${ }^{73}$. The $16 \mathrm{~S}$ rRNA gene sequencing was performed using a MiSeq (Illumina) at the DNA sequencing facility of GenProbio srl (www. genprobio.com $)^{73}$.

Following sequencing, the obtained individual sequence reads were filtered by the Illumina software to remove low quality and polyclonal sequences. All Illumina quality-approved, trimmed and filtered data were exported as.fastq files. The.fastq files were processed using a custom script based on the QIIME software suite ${ }^{74}$. Quality control retained sequences with a length between 140 and $400 \mathrm{bp}$ and mean sequence quality score $>20$ while sequences with homopolymers $>7$ bp and mismatched primers were omitted. To calculate downstream diversity measures (alpha and beta diversity indices, Unifrac analysis), 16S rRNA Operational Taxonomic Units (OTUs) were defined at $\geq 99 \%$ sequence homology using uclust $^{75}$ and OTUs with less than 10 sequences were filtered. All reads were classified to the lowest possible taxonomic rank using QIIME ${ }^{74}$ and a reference dataset from the SILVA database. Biodiversity of the samples (alpha-diversity) were calculated with Chaol and Shannon indexes. Similarities between samples (betadiversity) were calculated by unweighted uniFrac ${ }^{76}$. The range of similarities is calculated between the values 0 and 1 . PCoA representations of beta-diversity were performed using QIIME ${ }^{74}$

TNBS-induced experimental colitis. For TNBS-induced acute colitis, mice were rectally challenged with $3 \mathrm{mg}$ of Picrylsulfonic acid (TNBS, MW 293.17 Fluka). The weight curve was determined by weighing mice daily. At sacrifice colons were collected, their length was measured and divided in portions to be fixed in $10 \%$ 
formalin for histological analyses, snap-frozen for RNA extraction and for lamina propria mononuclear cells (LPMC) immunophenotyping.

Metabolomic analysis. Metabolome extraction, purification and derivatization was carried by means of the MetaboPrep kit (Theoreo srl, Montecorvino Pugliano [SA], Italy) according to the manufacturer's instruction.

Two $\mu \mathrm{L}$ samples of the derivatized solution were injected into the GC-MS system (GC-2010 Plus gas chromatograph coupled to a 2010 Plus single quadrupole mass spectrometer; Shimadzu Corp., Kyoto, Japan). Chromatographic separation was achieved with a $30 \mathrm{~m} 0.25 \mathrm{~mm}$ CP-Sil 8 CB fused silica capillary GC column with $1.00 \mu \mathrm{m}$ film thickness from Agilent (Agilent, J\&W Scientific, Folsom, CA, USA), with helium as carrier gas. The initial oven temperature of $100^{\circ} \mathrm{C}$ was maintained for $1 \mathrm{~min}$ and then raised by $4{ }^{\circ} \mathrm{C} / \mathrm{min}$ to $320^{\circ} \mathrm{C}$ with a further $4 \mathrm{~min}$ of hold time. The gas flow was set to obtain a constant linear velocity of $39 \mathrm{~cm} / \mathrm{s}$ and the split flow was set at 1:5. The mass spectrometer was operated in electron impact $(70 \mathrm{eV})$ in full scan mode in the interval of $35-600 \mathrm{~m} / \mathrm{z}$ with a scan velocity of 3333 $\mathrm{amu} / \mathrm{sec}$ and a solvent cut time of $4.5 \mathrm{~min}$. The complete GC program duration was $60 \mathrm{~min}$. Untargeted metabolites were identified by comparing the mass spectrum of each peak with the NIST library collection (NIST, Gaithersburg, MD, USA). To identify metabolites identity, the linear index difference max tolerance was set at 10 , while the minimum matching for the NIST library search was set at $85 \%$. The chromatographic data for PLS-DA analysis were tabulated with one sample per row and one variable (metabolite) per column. According to MSI level 1 standard ${ }^{77}$, the VIP putative metabolites identity was confirmed by means of an independent analytical standard analysis. The normalization procedures consisted of data transformation and scaling. Data transformation was made by generalized log transformation and data scaling by autoscaling (mean-centered and divided by standard deviation of each variable)

Metabolomics data analysis. Partial least square discriminant analysis (PLS-DA) ${ }^{78}$ was performed on Internal Standard peak area ${ }^{79}$ normalized chromatogram using R (Foundation for Statistical Computing, Vienna, Austria). Mean centering and unit variance scaling was applied for all analyses. Classes separation was archived by PLS-DA, which is a supervised method that uses multivariate regression techniques to extract, via linear combinations of original variables $(\mathrm{X})$, the information that can predict class membership (Y). PLS regression was performed using the plsr function included in the R pls package ${ }^{80}$. Classification and cross-validation was performed using the corresponding wrapper function included in the caret package. A permutation test was performed to assess the significance of class discrimination. In each permutation, a PLS-DA model was built between the data (X) and the permuted class labels $(\mathrm{Y})$ using the optimal number of components determined by cross validation for the model based on the original class assignment. Variable Importance in Projection (VIP) scores were calculated for each component. A VIP is a weighted sum of squares of the PLS loadings, taking into account the amount of explained Y-variation in each dimension.

To identify the most meaningful changes in two conditions, the volcano plot was used. This combines a measure of statistical significance from a statistical test ( $p$ value) with the magnitude of the change, enabling quick visual identification of those data points (metabolites) that display large magnitude changes that are also statistically significant. The volcano plots were constructed by plotting the negative $\log$ of the $p$ value on the $y$ axis. This results in data points with low $p$ values (highly significant) appearing toward the top of the plot. The $x$ axis was the log of the fold change between the two conditions. The log of the fold change is used so that changes in both directions appear equidistant from the center. Plotting points results in two regions of interest in the plot: those points that are found toward the top of the plot that are far to either the left- or right-hand sides. These represent values that display large magnitude fold changes (hence being left or right of center) as well as high statistical significance (hence being toward the top).

In vitro T-cell activation assay. Colonic lamina propria and mesenteric lymph node leukocytes were collected from untreated mice. $2 \times 10^{6}$ cells were plated and exposed to $0.05 \mathrm{mg}$ of bacteria (wet weight) derived from faeces of untreated, DSStreated or FMT-treated mice at the time of sacrifice. Gentamycin $(50 \mu \mathrm{g} / \mathrm{ml})$ and a cocktail of antibiotics $(\mathrm{P} / \mathrm{S})$ were added after $2 \mathrm{~h}$ of incubation. The cells were left in culture for $96 \mathrm{~h}$. In some experiments, anti-MHCII blocking antibody (clone M5/ 114.15.2, TONBO) was added at a final concentration of $10 \mu \mathrm{g} / \mathrm{ml}$. At the end of the experiment cells were analysed with flow cytometry. Their viability was checked with Zombie Yellow" Fixable Viability Kit (Biolegend).

Tissue ELISA of murine IL-10. Colonic tissues were homogenized in $300 \mu$ RIPA Buffer (Cell Signaling Technology) supplemented with Phosphatase inhibitors (Sigma) and Protease inhibitors (Complete Ultra tablets, Roche). The samples were then incubated at $4{ }^{\circ} \mathrm{C}$ for 30 min under slow rotation and then centrifuged at 13,000 r.p.m. $(16.2 \times g)$ for $15 \mathrm{~min}$ at $4^{\circ} \mathrm{C}$. The supernatant was quantified at the NanoDrop with Bradford Assay (BioRad). mIIL-10 was measured on $6.25 \mu \mathrm{g}$ of lysate using the ELISA assay (Purified anti-mouse IL-10 and Biotin anti-mouse IL10 , Biolegend) performed following manufacturer's instructions.
Faecal bacteria plating. One faecal pellet from each mouse was smashed, filtered with $100 \mu \mathrm{m}$ nylon cell strainer and resuspended in $1 \mathrm{~mL}$ of sterile PBS. A concentration of $200 \mu \mathrm{L}$ were then plated on Chocolate II Agar plates (BD) and grown at $37^{\circ} \mathrm{C}$ under aerobic conditions overnight or in anaerobic conditions for $48 \mathrm{~h}$.

Quantification and statistical analysis. Statistical analysis was performed with GraphPad Prism 5 (GraphPad Software). Statistical significance was calculated using a Mann-Whitney test for comparison within two groups or Kruskal-Wallis test with Dunn's multiple comparison correction within more than two groups. ${ }^{\star} P<0.05,{ }^{* \star} P<0.01,{ }^{* *} P<0.001$ were regarded as statistically significant. Outliers were detected with Grubb’s test. Non parametric distributions were represented as median $+/$ - interquartile range.

\section{Data availability}

$16 \mathrm{~S}$ rRNA raw data for Figs. 2a-f, 3a, b, 4d, f, 8b, e and Supplementary 4a, b, 5a, c, are available in SRA Online Repository associated to BioProject PRJNA494680.

Received: 4 January 2018 Accepted: 25 October 2018

Published online: 05 December 2018

\section{References}

1. Honda, K. \& Littman, D. R. The microbiota in adaptive immune homeostasis and disease. Nature 535, 75-84 (2016).

2. Kaser, A., Zeissig, S. \& Blumberg, R. S. Inflammatory bowel disease. Annu. Rev. Immunol. 28, 573-621 (2010).

3. Antharam, V. C. et al. Intestinal dysbiosis and depletion of butyrogenic bacteria in Clostridium difficile infection and nosocomial diarrhea. J. Clin. Microbiol. 51, 2884-2892 (2013).

4. Gevers, D. et al. The treatment-naive microbiome in new-onset Crohn's disease. Cell Host Microbe 15, 382-392 (2014).

5. Lepage, P. et al. Twin study indicates loss of interaction between microbiota and mucosa of patients with ulcerative colitis. Gastroenterology 141, 227-236 (2011).

6. Kostic, A. D. et al. Fusobacterium nucleatum potentiates intestinal tumorigenesis and modulates the tumor-immune microenvironment. Cell Host Microbe 14, 207-215 (2013).

7. Duchmann, R. et al. Tolerance exists towards resident intestinal flora but is broken in active inflammatory bowel disease (IBD). Clin. Exp. Immunol. 102 448-455 (1995).

8. Hedin, C. et al. Siblings of patients with Crohn's disease exhibit a biologically relevant dysbiosis in mucosal microbial metacommunities. Gut 65, 944-953 (2016).

9. Shah, R. et al. Composition and function of the pediatric colonic mucosal microbiome in untreated patients with ulcerative colitis. Gut Microbes 7, 384-396 (2016).

10. Ananthakrishnan, A. N. et al. Gut microbiome function predicts response to anti-integrin biologic therapy in inflammatory Bowel diseases. Cell Host Microbe 21, 603-610 e603 (2017)

11. Ivanov, I. I. et al. Induction of intestinal Th17 cells by segmented filamentous bacteria. Cell 139, 485-498 (2009).

12. Rescigno, M. The intestinal epithelial barrier in the control of homeostasis and immunity. Trends Immunol. 32, 256-264 (2011).

13. McIlroy, J., Ianiro, G., Mukhopadhya, I., Hansen, R. \& Hold, G. L. Review article: the gut microbiome in inflammatory bowel disease-avenues for microbial management. Aliment. Pharmacol. \& Ther. 47, 26-42 (2017).

14. Cammarota, G. et al. European consensus conference on faecal microbiota transplantation in clinical practice. Gut 66, 569-580 (2017).

15. van Nood, E. et al. Duodenal infusion of donor feces for recurrent Clostridium difficile. New Engl. J. Med. 368, 407-415 (2013).

16. Paramsothy, S. et al. Multidonor intensive faecal microbiota transplantation for active ulcerative colitis: a randomised placebo-controlled trial. Lancet 389, 1218-1228 (2017)

17. Rossen, N. G. et al. Findings from a randomized controlled trial of fecal transplantation for patients with ulcerative colitis. Gastroenterology 149, 110-118 e114 (2015).

18. Moayyedi, P. et al. Fecal microbiota transplantation induces remission in patients with active ulcerative colitis in a randomized controlled trial. Gastroenterology 149, 102-109 e106 (2015).

19. Tian, Z. et al. Beneficial effects of fecal microbiota transplantation on ulcerative colitis in mice. Dig. Dis. Sci. 61, 2262-2271 (2016).

20. Selvanantham, T. et al. NKT cell-deficient mice harbor an altered microbiota that fuels intestinal inflammation during chemically induced colitis. $J$. Immunol. 197, 4464-4472 (2016)

21. Caprioli, F., Pallone, F. \& Monteleone, G. Th17 immune response in IBD: new pathogenic mechanism. J. Crohn's \& Colitis 2, 291-295 (2008). 
22. Facciotti, F. et al. Peroxisome-derived lipids are self antigens that stimulate invariant natural killer T cells in the thymus. Nat. Immunol. 13, 474-480 (2012).

23. Tupin, E., Kinjo, Y. \& Kronenberg, M. The unique role of natural killer T cells in the response to microorganisms. Nat. Rev. Microbiol. 5, 405-417 (2007).

24. Olszak, T. et al. Microbial exposure during early life has persistent effects on natural killer T cell function. Science 336, 489-493 (2012).

25. An, D. et al. Sphingolipids from a symbiotic microbe regulate homeostasis of host intestinal natural killer T cells. Cell 156, 123-133 (2014).

26. Burrello, C. et al. Short-term oral antibiotics treatment promotes inflammatory activation of colonic invariant natural killer $\mathrm{T}$ and conventional CD4(+) T cells. Front. Med. 5, 21 (2018).

27. Geissmann, F. et al. Intravascular immune surveillance by CXCR6+NKT cells patrolling liver sinusoids. PLoS Biol. 3, e113 (2005).

28. Koon, H. W. et al. Cathelicidin signaling via the Toll-like receptor protects against colitis in mice. Gastroenterology 141, 1852-1863 (2011). e1851-1853.

29. Ueno, K. et al. MUC1 mucin is a negative regulator of toll-like receptor signaling. Am. J. Respir. Cell Mol. Biol. 38, 263-268 (2008).

30. Munyaka, P. M., Rabbi, M. F., Khafipour, E. \& Ghia, J. E. Acute dextran sulfate sodium (DSS)-induced colitis promotes gut microbial dysbiosis in mice. J. Basic Microbiol. 56, 986-998 (2016).

31. Osaka, T. et al. Meta-analysis of fecal microbiota and metabolites in experimental colitic mice during the inflammatory and healing phases. Nutrients 9, E1329 (2017).

32. Bibiloni, R. et al. VSL\#3 probiotic-mixture induces remission in patients with active ulcerative colitis. Am. J. Gastroenterol. 100, 1539-1546 (2005).

33. Palm, N. W. et al. Immunoglobulin A coating identifies colitogenic bacteria in inflammatory bowel disease. Cell 158, 1000-1010 (2014).

34. Michielan, A. \& D'Inca, R. Intestinal permeability in inflammatory bowel disease: pathogenesis, clinical evaluation, and therapy of leaky gut. Mediat. Inflamm. 2015, 628157 (2015).

35. Kolho, K. L., Pessia, A., Jaakkola, T., de Vos, W. M. \& Velagapudi, V. Faecal and serum metabolomics in paediatric inflammatory bowel disease. J. Crohn's \& Colitis 11, 321-334 (2017)

36. Sears, M. E. Chelation: harnessing and enhancing heavy metal detoxification-a review. Sci. World J. 2013, 219840 (2013).

37. Beloborodova, N. et al. Effect of phenolic acids of microbial origin on production of reactive oxygen species in mitochondria and neutrophils. J. Biomed. Sci. 19, 89 (2012).

38. Rooks, M. G. \& Garrett, W. S. Gut microbiota, metabolites and host immunity. Nat. Rev. Immunol. 16, 341-352 (2016).

39. Roy, U. et al. Distinct microbial communities trigger colitis development upon intestinal barrier damage via innate or adaptive immune cells. Cell Rep. 21, 994-1008 (2017)

40. Nozu, R., Ueno, M. \& Hayashimoto, N. Composition of fecal microbiota of laboratory mice derived from Japanese commercial breeders using $16 \mathrm{~S}$ rRNA gene clone libraries. J. Vet. Med. Sci. 78, 1045-1050 (2016).

41. Zhao, L. et al. Gut bacteria selectively promoted by dietary fibers alleviate type 2 diabetes. Science 359, 1151-1156 (2018).

42. Smith, P. M. et al. The microbial metabolites, short-chain fatty acids, regulate colonic Treg cell homeostasis. Science 341, 569-573 (2013)

43. Arpaia, N. et al. Metabolites produced by commensal bacteria promote peripheral regulatory T-cell generation. Nature 504, 451-455 (2013).

44. Furusawa, Y. et al. Commensal microbe-derived butyrate induces the differentiation of colonic regulatory T cells. Nature 504, 446-450 (2013).

45. Macpherson, A. J. \& Harris, N. L. Interactions between commensal intestinal bacteria and the immune system. Nat. Rev. Immunol. 4, 478-485 (2004).

46. Bernink, J. H. et al. Human type 1 innate lymphoid cells accumulate in inflamed mucosal tissues. Nat. Immunol. 14, 221-229 (2013).

47. Lim, A. I. et al. IL-12 drives functional plasticity of human group 2 innate lymphoid cells. J. Exp. Med. 213, 569-583 (2016)

48. Neurath, M. F. Cytokines in inflammatory bowel disease. Nat. Rev. Immunol. 14, 329-342 (2014)

49. Fung, T. C. et al. Lymphoid-tissue-resident commensal bacteria promote members of the il-10 cytokine family to establish mutualism. Immunity $\mathbf{4 4}$ 634-646 (2016).

50. Zackular, J. P., Baxter, N. T., Chen, G. Y. \& Schloss, P. D. Manipulation of the gut microbiota reveals role in colon tumorigenesis. mSphere 1, 00001 (2016).

51. Hand, T. W., Vujkovic-Cvijin, I., Ridaura, V. K. \& Belkaid, Y. Linking the microbiota, chronic disease, and the immune system. Trends Endocrinol. Metab.: TEM 27, 831-843 (2016).

52. Di Giacinto, C., Marinaro, M., Sanchez, M., Strober, W. \& Boirivant, M. Probiotics ameliorate recurrent Th1-mediated murine colitis by inducing IL10 and IL-10-dependent TGF-beta-bearing regulatory cells. J. Immunol. 174, 3237-3246 (2005).
53. Shaw, M. H., Kamada, N., Kim, Y. G. \& Nunez, G. Microbiota-induced IL1beta, but not IL-6, is critical for the development of steady-state TH17 cells in the intestine. J. Exp. Med. 209, 251-258 (2012).

54. Kostic, A. D., Xavier, R. J. \& Gevers, D. The microbiome in inflammatory bowel disease: current status and the future ahead. Gastroenterology 146 1489-1499 (2014).

55. Human Microbiome Project C. Structure, function and diversity of the healthy human microbiome. Nature 486, 207-214 (2012).

56. Nizzoli, G. et al. Pathogenicity of in vivo generated intestinal Th17 lymphocytes is IFNgamma dependent. J. Crohn's Colitis 12, 981-992 (2018).

57. Nistala, K. et al. Th17 plasticity in human autoimmune arthritis is driven by the inflammatory environment. Proc. Natl Acad. Sci. USA 107, 14751-14756 (2010).

58. Fujino, S. et al. Increased expression of interleukin 17 in inflammatory bowe disease. Gut 52, 65-70 (2003).

59. Duchmann, R. et al. T cell specificity and cross reactivity towards enterobacteria, bacteroides, bifidobacterium, and antigens from resident intestinal flora in humans. Gut 44, 812-818 (1999).

60. Yang, Y. et al. Focused specificity of intestinal TH17 cells towards commensal bacterial antigens. Nature 510, 152-156 (2014).

61. Nieuwenhuis, E. E. et al. Cd1d-dependent regulation of bacterial colonization in the intestine of mice. J. Clin. Investig. 119, 1241-1250 (2009).

62. Biancheri, P. et al. Absence of a role for interleukin-13 in inflammatory bowel disease. Eur. J. Immunol. 44, 370-385 (2014).

63. Huber, S. et al. Th17 cells express interleukin-10 receptor and are controlled by Foxp3(-) and Foxp3 + regulatory CD4 + T cells in an interleukin-10dependent manner. Immunity 34, 554-565 (2011)

64. Moore, K. W., de Waal Malefyt, R., Coffman, R. L. \& O’Garra, A. Interleukin10 and the interleukin-10 receptor. Annu. Rev. Immunol. 19, 683-765 (2001).

65. Duranti, S. et al. Elucidating the gut microbiome of ulcerative colitis: bifidobacteria as novel microbial biomarkers. FEMS Microbiol. Ecol. 92, fiw191 (2016)

66. Sag, D., Krause, P., Hedrick, C. C., Kronenberg, M. \& Wingender, G. IL-10producing NKT10 cells are a distinct regulatory invariant NKT cell subset. $J$. Clin. Investig. 124, 3725-3740 (2014)

67. Vos, A. C. et al. Anti-tumor necrosis factor-alpha antibodies induce regulatory macrophages in an Fc region-dependent manner. Gastroenterology 140, 221-230 (2011).

68. Fournier, B. M. \& Parkos, C. A. The role of neutrophils during intestinal inflammation. Mucosal Immunol. 5, 354-366 (2012).

69. Caprioli, F. et al. Reduction of CD68+macrophages and decreased IL-17 expression in intestinal mucosa of patients with inflammatory bowel disease strongly correlate with endoscopic response and mucosal healing following infliximab therapy. Inflamm. bowel Dis. 19, 729-739 (2013).

70. Fais, S. et al. HLA-DR antigens on colonic epithelial cells in inflammatory bowel disease: I. Relation to the state of activation of lamina propria lymphocytes and to the epithelial expression of other surface markers. Clin. Exp. Immunol. 68, 605-612 (1987).

71. Cribiù, F. M. et al. Implementation of an automated inclusion system for the histological analysis of murine tissue samples: a feasibility study in DSSinduced chronic colitis. Eur. J. Inflam. 16, 1-12 (2018).

72. Furet, J. P. et al. Comparative assessment of human and farm animal faecal microbiota using real-time quantitative PCR. FEMS Microbiol. Ecol. 68, 351-362 (2009).

73. Milani, C. et al. Assessing the fecal microbiota: an optimized ion torrent $16 \mathrm{~S}$ rRNA gene-based analysis protocol. PloS ONE 8, e68739 (2013).

74. Caporaso, J. G. et al. QIIME allows analysis of high-throughput community sequencing data. Nat. Methods 7, 335-336 (2010).

75. Edgar, R. C. Search and clustering orders of magnitude faster than BLAST. Bioinformatics 26, 2460-2461 (2010).

76. Lozupone, C. \& Knight, R. UniFrac: a new phylogenetic method for comparing microbial communities. Appl. Environ. Microbiol. 71, 8228-8235 (2005).

77. Sumner, L. W. et al. Proposed minimum reporting standards for chemical analysis Chemical Analysis Working Group (CAWG) Metabolomics Standards Initiative (MSI). Metab.: Off. J. Metab. Soc. 3, 211-221 (2007).

78. Wold, S., Sjöström, M. \& Eriksson, L. PLS-regression: a basic tool of chemometrics. PLS Methods 58, 109-130 (2001).

79. Sysi-Aho, M., Katajamaa, M., Yetukuri, L. \& Oresic, M. Normalization method for metabolomics data using optimal selection of multiple internal standards. BMC Bioinforma. 8, 93 (2007).

80. Mevik, B.-H., Wehrens, R. The pls Package: Principal Component and Partial Least Squares Regression in R. J. Stat. Softw. s1, (2007).

\section{Acknowledgements}

We thank the members of Prof. Rescigno's group for the invaluable scientific support, Erika Mileti for technical assistance during the revision, the IEO Animal Facility for the excellent animal husbandry and the NIH Tetramer Facility for providing mCD1d:PBS57 
Tetramers. This work was made possible through grants of AIRC (Start-Up 14378 to F.F.) and Fondazione IRCCS Policlinico Maggiore Milano $(5 \times 1000$ Research Award to F.C.).

\section{Author contributions}

C.B. performed experiments, analysed and interpreted the data; F.G., G.N., S.C. performed experiments; F.M.C., G.E., G.L. performed and interpreted histological analyses; J.T., A.C. performed and interpreted metabolomic analyses; S.Guglietta, S.B. revised the manuscript; S.Guglielmetti, V.T. grew bacteria; F.C., M.R. contributed to interpretation of the data and gave important intellectual contributions and revised the manuscript; F.F. designed, conceived and supervised the study and wrote the manuscript.

\section{Additional information}

Supplementary Information accompanies this paper at https://doi.org/10.1038/s41467018-07359-8.

Competing interests: The authors declare no competing interests

Reprints and permission information is available online at http://npg.nature.com/ reprintsandpermissions/
Publisher's note: Springer Nature remains neutral with regard to jurisdictional claims in published maps and institutional affiliations.

(c) (i) Open Access This article is licensed under a Creative Commons Attribution 4.0 International License, which permits use, sharing, adaptation, distribution and reproduction in any medium or format, as long as you give appropriate credit to the original author(s) and the source, provide a link to the Creative Commons license, and indicate if changes were made. The images or other third party material in this article are included in the article's Creative Commons license, unless indicated otherwise in a credit line to the material. If material is not included in the article's Creative Commons license and your intended use is not permitted by statutory regulation or exceeds the permitted use, you will need to obtain permission directly from the copyright holder. To view a copy of this license, visit http://creativecommons.org/ licenses/by/4.0/.

(C) The Author(s) 2018 